\title{
CALABI-YAU MANIFOLDS AND GENERIC HODGE GROUPS*
}

\author{
JAN CHRISTIAN ROHDE ${ }^{\dagger}$
}

\begin{abstract}
We study the generic Hodge groups $\operatorname{Hg}(\mathcal{X})$ of local universal deformations $\mathcal{X}$ of Calabi-Yau 3-manifolds with onedimensional complex moduli, give a complete list of all possible choices for $\operatorname{Hg}(\mathcal{X})_{\mathbb{R}}$ and determine the latter real groups for known examples.
\end{abstract}

Key words. Calabi-Yau manifold, variation of Hodge structures.

AMS subject classifications. 14D07.

Introduction. Let $X$ be a Calabi-Yau 3-manifold with $h^{2,1}(X)=1$. Moreover let $f: \mathcal{X} \rightarrow B$ denote the local universal deformation of $X$ and $Q$ denote the symplectic form on $H^{3}(X, \mathbb{Q})$ given by the cup product. In the generic Hodge group $\operatorname{Hg}(\mathcal{X})$ information about the arithmetic of the fibers, the variation of Hodge structures and the monodromy groups of the families containing $X$ as fiber is encoded. Similar computations are made for the Lie algebras of monodromy groups of families of $K 3$ surfaces in the appendix of [15]. Since the monodromy group $\operatorname{Mon}^{0}(\mathcal{X})$ is a normal subgroup of the derived Hodge group $\operatorname{Hg}^{\operatorname{der}}(\mathcal{X})$, this is related to our results. Here we classify the possible generic Hodge groups of $\mathcal{X}$, which is also a natural problem by itself.

In the case of a Calabi-Yau 3-manifold with $h^{2,1}(X)=1$ we consider a Hodge structure on $H^{3}(X, \mathbb{Q})$, which is a vector space of dimension 4 . We have much information about the variation of Hodge structures $(V H S)$ of families of Calabi-Yau 3-manifolds. For example by Bryant, Griffiths [2], we have a classical description of the $V H S$ of such families. By using the Hodge structure on $H^{3}(X, \mathbb{Q})$, one can construct the associated Weil- and the Griffiths intermediate Jacobians and their corresponding Hodge structures as introduced by C. Borcea [1]. These latter Hodge structures are given by the representations $h_{W}$ and $h_{G}$ of the circle group $S^{1}$ on $H^{3}(X, \mathbb{Q})$. In particular the centralizers $C\left(h_{G}(i)\right)$ and $C\left(h_{W}(i)\right)$ in $\operatorname{Sp}\left(H^{3}(X, \mathbb{R}), Q\right)$ will be helpful. By using these techniques, the theory of bounded symmetric domains [6], the theory of Shimura varieties [3], [4], [7], [9] and some intricate computations, we obtain the result:

TheOREM 0.1. Let $\mathcal{X}$ denote the local universal deformation of a Calabi-Yau 3-manifold $X$ with $h^{2,1}(X)=1$. Then one of the following cases holds true:

1.

$$
\operatorname{Hg}(\mathcal{X})=\operatorname{Sp}\left(H^{3}(X, \mathbb{Q}), Q\right)
$$

2.

$$
\operatorname{Hg}(\mathcal{X})_{\mathbb{R}}=C\left(h_{G}(i)\right)
$$

3. The representation of $\mathrm{Hg}(\mathcal{X})_{\mathbb{R}}$ on $H^{3}(X, \mathbb{R})$ is isomorphic to the natural representation of $\mathrm{SL}_{\mathbb{R}}(2)$ on $\operatorname{Sym}^{3}\left(\mathbb{R}^{2}\right)$.

\footnotetext{
*Received January 31, 2011; accepted for publication February 2, 2012.

$\dagger$ Department Mathematik, Universität Hamburg, Bundesstraße 55, 20146 Hamburg, Germany (jan.christian.rohde@math.uni-hamburg.de).
} 
In the case (2) we will also give an explicite description of $\operatorname{Hg}(\mathcal{X})_{\mathbb{R}}$, which tells us that one has a reducible representation in this case. At present there does not exist any example of a family of Calabi-Yau 3-manifolds known to the author, which has a generic Hodge group satisfying (3). Nevertheless we will determine the generic Hodge groups of known examples of Calabi-Yau 3-manifolds and see that there exists a Calabi-Yau like variation of Hodge structures satisfying (3).

1. Facts and conventions. Here a Calabi-Yau 3-manifold $X$ is a compact Kähler manifold of complex dimension 3 such that

$$
H^{1,0}(X)=H^{2,0}(X)=0 \text { and } \omega_{X} \cong \mathcal{O}_{X}
$$

We will only study Calabi-Yau 3-manifolds $X$ with $h^{2,1}(X)=1$ here. Let $f: \mathcal{X} \rightarrow B$ denote the local universal deformation of $X \cong \mathcal{X}_{0}$, where $0 \in B$.

Moreover recall the algebraic groups

$$
S^{1}=\operatorname{Spec}\left(\mathbb{R}[x, y] / x^{2}+y^{2}-1\right) \text { and } \mathbb{S}=\operatorname{Spec}\left(\mathbb{R}[t, x, y] / t\left(x^{2}+y^{2}\right)-1\right),
$$

where

$$
S^{1}(\mathbb{R})=\left\{M=\left(\begin{array}{cc}
a & b \\
-b & a
\end{array}\right) \in \mathrm{SL}_{2}(\mathbb{R})\right\} \cong\{z \in \mathbb{C}:|z|=1\}
$$

and

$$
\mathbb{S}(\mathbb{R})=\left\{\left(\begin{array}{cc}
a & b \\
-b & a
\end{array}\right) \in \mathrm{GL}_{2}(\mathbb{R})\right\} \cong \mathbb{C}^{*}
$$

The group $\mathbb{S}$ is the Deligne torus given by the Weil restriction $R_{\mathbb{C} / \mathbb{R}}\left(\mathbb{G}_{m}\right)$ and $S^{1}$ is a subgroup of $\mathbb{S}$. Let $V$ be a real vector space. By the eigenspace decompositions of $V_{\mathbb{C}}$ with respect to the characters $z^{p} \bar{z}^{q}$ for $p, q \in \mathbb{Z}$ of $\mathbb{S}$, the real representations $h: \mathbb{S} \rightarrow \operatorname{GL}(V)$ correspond to the Hodge structures on $V$ (see [4], 1.1.1). If there is some fixed $k$ such that all characters $z^{p} \bar{z}^{q}$ with non-trivial associated eigenspace satisfy $p+q=k$, one says that the Hodge structure has weight $k$. There exists an embedding $w: \mathbb{G}_{m, \mathbb{R}} \hookrightarrow \mathbb{S}$ given by

$$
\mathbb{G}_{m}(\mathbb{R}) \cong\left\{\operatorname{diag}(a, a) \in \mathrm{GL}_{2}(\mathbb{R})\right\} \stackrel{\mathrm{id}}{\hookrightarrow} \mathbb{S}(\mathbb{R}) .
$$

The Hodge structure $h$ has weight $k$, if and only if the weight homomorphism $h \circ w$ satisfies

$$
r \rightarrow \operatorname{diag}\left(r^{k}, \ldots, r^{k}\right) \quad\left(\forall r \in \mathbb{R}^{*}=\mathbb{G}_{m}(\mathbb{R})\right)
$$

(see [10], Remark 1.1.4). Hodge structures of some given weight $k$ are determined by the restricted representation $\left.h\right|_{S^{1}}$. For example the integral Hodge structure on $H^{3}(X, \mathbb{Z})$ of weight 3 corresponds to the representation

$$
h_{X}: S^{1} \rightarrow \operatorname{GL}\left(H^{3}(X, \mathbb{R})\right), \quad h_{X}(z) v=z^{p} \bar{z}^{q} v \quad\left(\forall v \in H^{p, q}(X) \text { with } p+q=3\right) .
$$

We also denote $h_{X}$ by $h$ for short. The Hodge group $\operatorname{Hg}\left(H^{3}(X, \mathbb{Q}), h\right) \subset$ $\operatorname{GL}\left(H^{3}(X, \mathbb{Q})\right)$ is the smallest $\mathbb{Q}$-algebraic group $G \subset \mathrm{GL}\left(H^{3}(X, \mathbb{Q})\right)$ with $h\left(S^{1}\right) \subset$ $G_{\mathbb{R}}$. Assume without loss of generality that $B$ is contractible. Thus for each $b \in B$ one has a canonical isomorphism

$$
H^{3}\left(\mathcal{X}_{b}, \mathbb{Q}\right) \cong R^{3} f_{*}(\mathbb{Q})(B) \cong H^{3}\left(\mathcal{X}_{0}, \mathbb{Q}\right)=H^{3}(X, \mathbb{Q})
$$


By using this isomorphism, a subgroup of $\mathrm{GL}\left(H^{3}\left(\mathcal{X}_{b}, \mathbb{Q}\right)\right)$ can be considered as a subgroup of $\mathrm{GL}\left(H^{3}(X, \mathbb{Q})\right)$. This allows to define an inclusion relation for the Hodge groups of the several fibers, which we use now. The generic Hodge group $\operatorname{Hg}(\mathcal{X})$ of $\mathcal{X}$ is given by the generic Hodge group of the rational variation of Hodge structures $(V H S)$ of weight 3 of $\mathcal{X}$. Recall that the generic Hodge group of a $V H S$ is the maximum of the Hodge groups of all occurring Hodge structures. In an analogue way one can define the Mumford-Tate group $\operatorname{MT}\left(H^{3}(X, \mathbb{Q}), h\right)$ and the generic Mumford-Tate group $\operatorname{MT}(\mathcal{X})$ by using $h(\mathbb{S})$ instead of $h\left(S^{1}\right)$. One has that $\operatorname{MT}\left(H^{3}\left(\mathcal{X}_{b}, \mathbb{Q}\right), h_{b}\right)=\operatorname{MT}(\mathcal{X})$ over the complement of countably many proper analytic subsets of the basis (follows from [9], 1.2). Since

$$
\operatorname{Hg}\left(H^{3}\left(\mathcal{X}_{b}, \mathbb{Q}\right), h\right)=\left(\operatorname{MT}\left(H^{3}\left(\mathcal{X}_{b}, \mathbb{Q}\right), h\right) \cap \operatorname{SL}\left(H^{3}\left(\mathcal{X}_{b}, \mathbb{Q}\right)\right)\right)^{0}
$$

(see [10], Lemma 1.3.17), one has also that $\operatorname{Hg}\left(H^{3}\left(\mathcal{X}_{b}, \mathbb{Q}\right), h_{b}\right)=\operatorname{Hg}(\mathcal{X})$ over the complement of countably many proper analytic subsets of the basis.

1.1. We consider only algebraic groups over fields $K$ of characteristic zero. A group $G$ over $K$ is a torus, if $G_{\bar{K}} \cong \mathbb{G}_{m, \bar{K}}^{\ell}$. Moreover a group $G$ is simple, if it does not contain any proper connected normal subgroup. We say that $G$ is semisimple, if its maximal connected normal solvable subgroup is trivial.

A group $G$ is reductive, if it is the almost direct product of a torus and a semisimple group. In this situation the torus can be given by the connected component of identity of the center $Z(G)$ of $G$ and the semisimple group can be given by the derived subgroup $G^{\text {der }}$ generated by the commutators (follows from [12], page 9).

Let ad denote the adjoint representation. For a reductive group $G$, we have the exact sequence

$$
1 \rightarrow Z(G) \rightarrow G \rightarrow G^{\text {ad }} \rightarrow 1
$$

and the adjoint group $G^{\text {ad }}$ and $G^{\text {der }}$ are isogenous.

We say that a semisimple group is adjoint, if its center is trivial. It is a wellknown fact that connected semisimple adjoint $\mathbb{R}$-algebraic groups are direct products of simple subgroups.

It is a well-known fact that $\operatorname{Hg}(\mathcal{X})_{\mathbb{R}}^{0}$ and $\operatorname{MT}(\mathcal{X})_{\mathbb{R}}^{0}$ is defined over $\mathbb{Q}$. Moreover

$$
h\left(S^{1}\right) \subset \operatorname{Hg}(\mathcal{X})_{\mathbb{R}}^{0} \text { and } h(\mathbb{S}) \subset \operatorname{MT}(\mathcal{X})_{\mathbb{R}}^{0}
$$

Thus

$$
\operatorname{Hg}(\mathcal{X}), \quad \operatorname{Hg}(\mathcal{X})_{\mathbb{R}}, \quad \operatorname{MT}(\mathcal{X}) \text { and } \operatorname{MT}(\mathcal{X})_{\mathbb{R}}
$$

are Zariski connected. Moreover Hodge groups and Mumford-Tate groups of polarized rational Hodge structures are reductive (for example see [10], Theorem 1.3.16 and Corollary 1.3.20). From this fact and the definition of reductive groups one concludes that

$$
\operatorname{Hg}^{\operatorname{der}}(\mathcal{X})_{\mathbb{R}}, \quad \operatorname{Hg}^{\text {ad }}(\mathcal{X})_{\mathbb{R}}, \quad \operatorname{MT}^{\text {der }}(\mathcal{X})_{\mathbb{R}} \text { and } \operatorname{MT}^{\text {ad }}(\mathcal{X})_{\mathbb{R}}
$$

are also Zariski connected.

By knowing the associated Lie groups of $\mathbb{R}$-valued points, one can determine the isomorphism classes of some algebraic groups of our interest: 
Lemma 1.2. Assume that $G$ and $H$ are $\mathbb{R}$-algebraic connected semisimple adjoint groups, where $H(\mathbb{R})$ is a connected Lie group. Moreover let $h: G(\mathbb{R})^{+} \rightarrow H(\mathbb{R})$ be an isomorphism of Lie groups. Then $G$ and $H$ are isomorphic as $\mathbb{R}$-algebraic groups.

Proof. From the assumptions we conclude that there is an isomorphism $d h_{\mathbb{C}}$ : $\mathfrak{g}_{\mathbb{C}} \rightarrow \mathfrak{h}_{\mathbb{C}}$. Note that $\mathfrak{g}_{\mathbb{C}}$ and $\mathfrak{h}_{\mathbb{C}}$ are also semisimple as real Lie algebras and that for an arbitrary real Lie algebra $\mathfrak{g}^{\prime}$ one can define its adjoint Lie group $\operatorname{Int}\left(\mathfrak{g}^{\prime}\right)$ (see [6], II. §5). Due to the assumption that $G$ and $H$ are semisimple adjoint, the adjoint representation yields isomorphisms

$$
G(\mathbb{C})^{+} \cong \operatorname{Int}\left(\mathfrak{g}_{\mathbb{C}}\right) \text { and } H(\mathbb{C})^{+} \cong \operatorname{Int}\left(\mathfrak{h}_{\mathbb{C}}\right)
$$

Moreover for a real semisimple Lie algebra $\mathfrak{g}^{\prime}$ the connected component of identity of the Lie group given by the automorphism group of $\mathfrak{g}^{\prime}$ coincides with $\operatorname{Int}\left(\mathfrak{g}^{\prime}\right)$ (see [6], II. Corollary 6.5). Thus one concludes that $G(\mathbb{C})^{+}$and $H(\mathbb{C})^{+}$are the connected components of identity of the Lie groups given by the automorphism groups of $\mathfrak{g}_{\mathbb{C}}$ and $\mathfrak{h}_{\mathbb{C}}$. Therefore one obtains a holomorphic isomorphism $h_{\mathbb{C}}: G(\mathbb{C})^{+} \rightarrow H(\mathbb{C})^{+}$. By [12], I. Proposition 3.5, the semisimple Lie groups $G(\mathbb{C})^{+}$and $H(\mathbb{C})^{+}$are the groups of $\mathbb{C}$-valued points of $\mathbb{C}$-algebraic groups and the homomorphism $h_{\mathbb{C}}$ is a $\mathbb{C}$ algebraic regular map given by some polynomials $f_{1}, \ldots, f_{k}$ over $\mathbb{C}$. Since $\left.h_{\mathbb{C}}\right|_{G(\mathbb{R})^{+}}$ coincides with $h: G(\mathbb{R})^{+} \rightarrow H(\mathbb{R})$, one concludes that $\Im f_{1}, \ldots, \Im f_{k}$ vanish on the Zariski closure of $G(\mathbb{R})^{+}$. The Zariski closure of $G(\mathbb{R})^{+}$is $G$, since we assume that $G$ is Zariski connected. Thus the isomorphism $h$ is $\mathbb{R}$-algebraic.

1.3. Let $G$ be a connected $\mathbb{R}$-algebraic group and $\theta$ be an involutive automorphism of $G$. We say that $\theta$ is a Cartan involution, if the Lie subgroup

$$
G^{\theta}(\mathbb{R})=\{g \in G(\mathbb{C}) \mid g=\theta(\bar{g})\}
$$

of $G(\mathbb{C})$ is compact. An $\mathbb{R}$-algebraic group $G$ has a Cartan involution, if and only if $G$ is reductive (see [10], Proposition 1.3.10). In the case of a compact connected $\mathbb{R}$-algebraic group $K$ we have the Cartan involution $\operatorname{id}_{K}$ (see [10], Example 1.3.11). Thus all compact connected $\mathbb{R}$-algebraic groups are reductive.

The Griffiths intermediate Jacobian $J_{G}$ resp., the Weil intermediate Jacobian $J_{W}$ is the torus corresponding to the weight 1 Hodge structure given by

$$
F_{G}^{1}\left(H^{3}(X, \mathbb{C})\right)=F^{2}\left(H^{3}(X, \mathbb{C})\right) \text { resp., } \quad F_{W}^{1}\left(H^{3}(X, \mathbb{C})\right)=H^{3,0}(X) \oplus H^{1,2}(X){ }^{1}
$$

Let $h_{G}: S^{1} \rightarrow \mathrm{GL}\left(H^{3}(X, \mathbb{R})\right)$ and $h_{W}: S^{1} \rightarrow \mathrm{GL}\left(H^{3}(X, \mathbb{R})\right)$ denote the corresponding representations. It is a well-known fact that weight 1 Hodge structures correspond to complex structures. We will use the complex structures

$$
h_{G}(i) \text { and } h_{W}(i)=-h_{X}(i) .
$$

Moreover $h_{W}(z)$ and $h_{G}(z)$ commute and

$$
h(z)=h_{G}^{2}(z) h_{W}(z)
$$

\footnotetext{
${ }^{1}$ Note that in $[1]$ one has

$F_{W}^{1}\left(H^{3}(X, \mathbb{C})\right)=H^{0,3}(X) \oplus H^{2,1}(X)$ instead of $F_{W}^{1}\left(H^{3}(X, \mathbb{C})\right)=H^{3,0}(X) \oplus H^{1,2}(X)$.
}

But this is only a matter of the chosen conventions and personal preferences. 
Let $Q$ denote the symplectic form on $H^{3}(X, \mathbb{Q})$ given by the cup product. For the rest of this article let us fix $v_{p, 3-p} \in H^{p, 3-p}(X) \backslash\{0\}$ with

$$
\bar{v}_{p, 3-p}=v_{3-p, p} \text { and } Q\left(i v_{3,0}, v_{0,3}\right)=Q\left(-i v_{2,1}, v_{1,2}\right)=1 \text {. }
$$

There exist unique vectors satisfying these properties because of the well-known form of the polarization of $H^{3}(X, \mathbb{C})$ (see [14], 7.1.2) and the given Hodge numbers in our case. Thus our alternating form $Q$ on $H^{3}(X, \mathbb{C})$ is given by

$$
Q\left(\left(\begin{array}{l}
v_{1} \\
v_{2} \\
v_{3} \\
v_{4}
\end{array}\right),\left(\begin{array}{l}
w_{1} \\
w_{2} \\
w_{3} \\
w_{4}
\end{array}\right)\right)=\left(v_{1}, v_{2}, v_{3}, v_{4}\right)\left(\begin{array}{cccc}
0 & 0 & 0 & -i \\
0 & 0 & -i & 0 \\
0 & i & 0 & 0 \\
i & 0 & 0 & 0
\end{array}\right)\left(\begin{array}{l}
w_{1} \\
w_{2} \\
w_{3} \\
w_{4}
\end{array}\right)
$$

with respect to the basis $\left\{v_{3,0}, v_{1,2}, v_{2,1}, v_{0,3}\right\}$.

The reader can easily check that each $M \in \mathrm{GL}\left(H^{3}(X, \mathbb{R})\right)$ is given by a matrix

$$
M=\left(\begin{array}{llll}
v_{1} & w_{1} & \bar{w}_{4} & \bar{v}_{4} \\
v_{2} & w_{2} & \bar{w}_{3} & \bar{v}_{3} \\
v_{3} & w_{3} & \bar{w}_{2} & \bar{v}_{2} \\
v_{4} & w_{4} & \bar{w}_{1} & \bar{v}_{1}
\end{array}\right) \text {, where } v_{1}, \ldots, v_{4}, w_{1}, \ldots, w_{4} \in \mathbb{C}
$$

with respect to the basis $\left\{v_{3,0}, v_{2,1}, v_{1,2}, v_{0,3}\right\}$ by using the $\mathbb{R}$-vector space isomorphism given by the trace map

$$
F^{2}\left(H^{3}(X, \mathbb{C})\right) \rightarrow H^{3}(X, \mathbb{R}), \quad w \rightarrow w+\bar{w} .
$$

In a similar way on can easily check that the matrices with complex entries, which will occur in this paper, are in fact real.

REMARK 1.4. The conjugation by elements of $h_{X}\left(S^{1}\right)(\mathbb{R})$ is given by

$$
\begin{aligned}
& \left(\begin{array}{cccc}
\xi^{3} & 0 & 0 & 0 \\
0 & \xi & 0 & 0 \\
0 & 0 & \bar{\xi} & 0 \\
0 & 0 & 0 & \bar{\xi}^{3}
\end{array}\right)\left(\begin{array}{cccc}
a_{1,1} & a_{1,2} & a_{1,3} & a_{1,4} \\
a_{2,1} & a_{2,2} & a_{2,3} & a_{2,4} \\
a_{3,1} & a_{3,2} & a_{3,3} & a_{3,4} \\
a_{4,1} & a_{4,2} & a_{4,3} & a_{4,4}
\end{array}\right)\left(\begin{array}{cccc}
\bar{\xi}^{3} & 0 & 0 & 0 \\
0 & \bar{\xi} & 0 & 0 \\
0 & 0 & \xi & 0 \\
0 & 0 & 0 & \xi^{3}
\end{array}\right) \\
& =\left(\begin{array}{cccc}
a_{1,1} & \xi^{2} a_{1,2} & \xi^{4} a_{1,3} & \xi^{6} a_{1,4} \\
\bar{\xi}^{2} a_{2,1} & a_{2,2} & \xi^{2} a_{2,3} & \xi^{4} a_{2,4} \\
\bar{\xi}^{4} a_{3,1} & \bar{\xi}^{2} a_{3,2} & a_{3,3} & \bar{\xi}^{2} a_{3,4} \\
\bar{\xi}^{6} a_{4,1} & \bar{\xi}^{4} a_{4,2} & \bar{\xi}^{2} a_{4,3} & a_{4,4}
\end{array}\right)
\end{aligned}
$$

with respect to the basis $\left\{v_{3,0}, v_{2,1}, v_{1,2}, v_{0,3}\right\}$. Moreover the conjugation by the elements of $h_{W}\left(S^{1}(\mathbb{R})\right)$ is given by:

$$
\left(\begin{array}{llll}
\xi & 0 & 0 & 0 \\
0 & \bar{\xi} & 0 & 0 \\
0 & 0 & \xi & 0 \\
0 & 0 & 0 & \bar{\xi}
\end{array}\right)\left(\begin{array}{llll}
a_{1,1} & a_{1,2} & a_{1,3} & a_{1,4} \\
a_{2,1} & a_{2,2} & a_{2,3} & a_{2,4} \\
a_{3,1} & a_{3,2} & a_{3,3} & a_{3,4} \\
a_{4,1} & a_{4,2} & a_{4,3} & a_{4,4}
\end{array}\right)\left(\begin{array}{cccc}
\bar{\xi} & 0 & 0 & 0 \\
0 & \bar{\xi} & 0 & 0 \\
0 & 0 & \bar{\xi} & 0 \\
0 & 0 & 0 & \xi
\end{array}\right)\left(\begin{array}{cccc}
a_{1,1} & \xi^{2} a_{1,2} & a_{1,3} & \xi^{2} a_{1,4} \\
\bar{\xi}^{2} a_{2,1} & a_{2,2} & \bar{\xi}^{2} a_{2,3} & a_{2,4} \\
a_{3,1} & \xi^{2} a_{3,2} & a_{3,3} & \xi^{2} a_{3,4} \\
\bar{\xi}^{2} a_{4,1} & a_{4,2} & \bar{\xi}^{2} a_{4,3} & a_{4,4}
\end{array}\right)
$$

REMARK 1.5. The centralizer $C\left(h\left(S^{1}\right)\right)$ of $h\left(S^{1}\right)$ in $\operatorname{Sp}\left(H^{3}(X, \mathbb{R}), Q\right)$ is given by matrices $\operatorname{diag}(\xi, \zeta, \bar{\zeta}, \bar{\xi})$ with respect to the basis $\left\{v_{3,0}, v_{2,1}, v_{1,2}, v_{0,3}\right\}$ as one concludes by the description of the conjugation by elements of $h\left(S^{1}\right)(\mathbb{R})$ in Remark 1.4. Moreover by explicit computations using (1), one concludes $|\xi|=|\zeta|=1$. Thus 
$C\left(h\left(S^{1}\right)\right) \cong S^{1} \times S^{1}$. The group of real symplectic automorphisms in $C\left(h\left(S^{1}\right)\right)$, whose order is atmost 4 , is generated by $\operatorname{diag}(1, i,-i, 1)$ and $\operatorname{diag}(i, 1,1,-i)$. Thus $C\left(h\left(S^{1}\right)\right)$ contains only the complex structures

$$
\pm h_{W}(i)= \pm \operatorname{diag}(i,-i, i,-i) \text { and } \pm h_{G}(i)= \pm \operatorname{diag}(i, i,-i,-i) .
$$

Moreover $C\left(h\left(S^{1}\right)\right)$ is generated by $h_{W}\left(S^{1}\right)$ and $h_{G}\left(S^{1}\right)$. The kernel of the natural homomorphism

$$
h_{W}\left(S^{1}\right) \times h_{G}\left(S^{1}\right) \rightarrow C(h)
$$

obtained from multiplication is given by $\{(1,1),(-1,-1)\}$.

Let $C\left(h_{G}(i)\right)$ and $C\left(h_{W}(i)\right)$ denote the respective centralizers of $h_{G}(i)$ and $h_{W}(i)$ in $\operatorname{Sp}\left(H^{3}(X, \mathbb{R}), Q\right)$. The centralizer $C(h(i))$ of $h(i)$ in $\operatorname{Sp}\left(H^{3}(X, \mathbb{R}), Q\right)$ coincides with $C\left(h_{W}(i)\right)$, since $h_{W}(i)=-h(i)$. Let $H$ denote the Hermitian form

$$
H=i Q(\cdot, \cdot)
$$

Since $h(i)$ is a Hodge isometry of the real Hodge structure on $H^{3}(X, \mathbb{R})$, one concludes from the definition of $H$ as in [10], Section 4.3 and [11], Lemma 3.4:

Proposition 1.6. The group $C\left(h_{G}(i)\right)$ is given by $\operatorname{diag}(M, \bar{M})$, where

$$
M \in \mathrm{U}\left(F^{2}(X),\left.H\right|_{F^{2}(X)}\right)(\mathbb{R}) \cong \mathrm{U}(1,1)(\mathbb{R})
$$

and $\bar{M}$ acts on $\bar{F}^{2}(X)$.

In an analogue way one concludes: ${ }^{2}$

Proposition 1.7. The group $C\left(h_{W}(i)\right)$ is given by $\operatorname{diag}(M, \bar{M})$, where

$$
M \in \mathrm{U}\left(F^{2}(X),\left.H\right|_{H^{3,0}(X) \oplus H^{1,2}(X)}\right)(\mathbb{R}) \cong \mathrm{U}(2)(\mathbb{R})
$$

and $\bar{M}$ acts on $H^{0,3}(X) \oplus H^{2,1}(X)$.

Thus the unitary groups $\mathrm{U}(1,1)$ and $\mathrm{U}(2)$ will be important:

Remark 1.8. One can describe $\mathrm{U}(1,1)$ and $\mathrm{U}(2)$ explicitly. The special unitary group $\mathrm{SU}(1,1)$ is given by the matrices

$$
M_{1}=\left(\begin{array}{cc}
\alpha & \beta \\
\bar{\beta} & \bar{\alpha}
\end{array}\right) \text { with }|\alpha|^{2}-|\beta|^{2}=1 \text { and } \alpha, \beta \in \mathbb{C}
$$

(see [12], page 59) and $\mathrm{SU}(2)$ is given by the matrices

$$
M_{2}=\left(\begin{array}{cc}
\alpha & \beta \\
-\bar{\beta} & \bar{\alpha}
\end{array}\right) \text { with }|\alpha|^{2}-|\beta|^{2}=1 \text { and } \alpha, \beta \in \mathbb{C}
$$

as one concludes from the fact that $\bar{M}_{2}^{t}=M_{2}^{-1}$. Since

$$
\bar{M}_{1}^{t}\left(\begin{array}{cc}
1 & 0 \\
0 & -1
\end{array}\right) M_{1}=\left(\begin{array}{cc}
1 & 0 \\
0 & -1
\end{array}\right) \text { for } M_{1} \in \mathrm{U}(1,1)(\mathbb{R})
$$

\footnotetext{
${ }^{2}$ It should be pretty clear to the experts that the conjugacy class of $h_{W}\left(S^{1}\right)$ in $\operatorname{Sp}\left(H^{3}(X, \mathbb{Q}), Q\right)$ yields the upper half plane $\mathfrak{h}_{2}$, which is also a way to conclude that $C\left(h_{W}(i)\right) \cong \mathrm{U}(2)$.
} 
one has $\left|\operatorname{det} M_{1}\right|=1$. Thus the reductive group $\mathrm{U}(1,1)$ is the almost direct product of the simple group $\mathrm{SU}(1,1)$ and its center $Z(\mathrm{U}(1,1))$ isomorphic to $S^{1}$, where

$$
\mathrm{SU}(1,1) \cap Z(\mathrm{U}(1,1)) \cong\{ \pm 1\} .
$$

Moreover recall that

$$
\mathrm{SU}(1,1) \cong \mathrm{Sp}_{\mathbb{R}}(2) \cong \mathrm{SL}_{\mathbb{R}}(2)
$$

In an analogue way one concludes that the reductive group $\mathrm{U}(2)$ is the almost direct product of the simple group $\mathrm{SU}(2)$ and its center $Z(\mathrm{U}(2))$ isomorphic to $S^{1}$, where

$$
\mathrm{SU}(2) \cap Z(\mathrm{U}(2)) \cong\{ \pm 1\} .
$$

Moreover we will need an explicit description of the Lie algebra of $\mathrm{SU}(1,1)$ :

REMARK 1.9. One has that

$$
M_{1}=\left(\begin{array}{cc}
a & b \\
\bar{b} & \bar{a}
\end{array}\right) \in \mathrm{SU}(1,1)(\mathbb{R})
$$

is unipotent, if and only if

$$
2 \Re(a)=\operatorname{tr}\left(M_{1}\right)=2 .
$$

Since each nontrivial unipotent $M_{1} \in \mathrm{SU}(1,1)(\mathbb{R})$ has only one Jordan block of length 2 , one computes that

$$
\log M_{1}=M_{1}-E_{2}=\left(\begin{array}{cc}
i \Im(a) & b \\
\bar{b} & -i \Im(a)
\end{array}\right) .
$$

This yields 2 linearly independent vectors of $\mathfrak{s u}(1,1)$ given by $\log \left(M_{1}\right)=M_{1}-E_{2}$ for some unipotent $M_{1}$. By appending

$$
\log (\operatorname{diag}(a, \bar{a}))=\operatorname{diag}(i y,-i y)
$$

for $|a|=1$ and $y \in \mathbb{R}$, one obtains a basis of the threedimensional algebra $\mathfrak{s u}(1,1)$. Thus for each $N \in \mathfrak{s u}(1,1)(\mathbb{R})$ there are suitable $u, v, y \in \mathbb{R}$ such that

$$
N=\left(\begin{array}{cc}
i y & u+i v \\
u-i v & -i y
\end{array}\right) \text {. }
$$

REMARK 1.10. Since the centralizer $C\left(h_{G}(i)\right) \cong \mathrm{U}(1,1)$ of $h_{G}(i)$ is not compact, the conjugation by $h_{G}(i)$ does not yield a Cartan involution of $\operatorname{Sp}\left(H^{3}(X, \mathbb{R}), Q\right)$.

LEMma 1.11. The conjugation by $h_{W}(i)$ and the conjugation by $h_{X}(i)$ yield the same Cartan involutions on $\operatorname{Hg}(\mathcal{X})_{\mathbb{R}}$ resp., $\operatorname{Hg}^{\mathrm{der}}(\mathcal{X})_{\mathbb{R}}$. The conjugation by $\operatorname{ad}\left(h_{W}(i)\right)$ yields a Cartan involution on $\mathrm{Hg}^{\text {ad }}(\mathcal{X})_{\mathbb{R}}$.

Proof. Note that the conjugation by a complex structure

$$
J \in \operatorname{Sp}\left(H^{3}(X, \mathbb{R}), Q\right)(\mathbb{R}) \text { with } Q(J \cdot, \cdot)>0
$$


yields a Cartan involution of $\operatorname{Sp}\left(H^{3}(X, \mathbb{R}), Q\right)$ (see [7], page 67$)$. Since $Q\left(h_{W}(i) \cdot, \cdot\right)>$ 0 as one can verify by using (1) and (2), the conjugation by $h_{W}(i)$ yields a Cartan involution of $\operatorname{Sp}\left(H^{3}(X, \mathbb{R}), Q\right)(\mathbb{R})$. Due to the fact that $h_{W}(i) \in \operatorname{Hg}(\mathcal{X})_{\mathbb{R}}$, the conjugation by $h_{W}(i)$ yields a Cartan involution of the subgroup $\operatorname{Hg}(\mathcal{X})_{\mathbb{R}} \subset \operatorname{Sp}\left(H^{3}(X, \mathbb{R}), Q\right)$ (follows from [12], I. Theorem 4.2). Since $h_{W}(i)=-h_{X}(i)$, the conjugation by $h_{X}(i)$ yields the same involution.

Due to the fact that the reductive group $\operatorname{Hg}(\mathcal{X})_{\mathbb{R}}$ is the almost direct product of $Z(\operatorname{Hg}(\mathcal{X}))^{0}$ and its derived group $\operatorname{Hg}^{\text {der }}(\mathcal{X})_{\mathbb{R}}$, one concludes $h_{W}(i)=J_{C} \cdot J_{D}$, where $J_{C} \in Z(\operatorname{Hg}(\mathcal{X}))(\mathbb{C})^{0}$ and $J_{D} \in \operatorname{Hg}^{\operatorname{der}}(\mathcal{X})(\mathbb{C})$. Thus

$$
\begin{aligned}
h_{W}(i) \mathrm{Hg}^{\operatorname{der}}(\mathcal{X})(\mathbb{R}) h_{W}(i)^{-1} & =J_{C} J_{D} \operatorname{Hg}^{\operatorname{der}}(\mathcal{X})(\mathbb{R}) J_{D}^{-1} J_{C}^{-1} \\
& =J_{C} J_{C}^{-1} J_{D} \operatorname{Hg}^{\text {der }}(\mathcal{X})(\mathbb{R}) J_{D}^{-1} \\
& =J_{D} \operatorname{Hg}^{\operatorname{der}}(\mathcal{X})(\mathbb{R}) J_{D}^{-1}=\operatorname{Hg}^{\operatorname{der}}(\mathcal{X})(\mathbb{R}) .
\end{aligned}
$$

Therefore the conjugation by $h_{W}(i)$ yields a Cartan involution of $\operatorname{Hg}^{\text {der }}(\mathcal{X})_{\mathbb{R}}$. This Cartan involution corresponds clearly to a Cartan involution on $\mathrm{Hg}^{\text {ad }}(\mathcal{X})_{\mathbb{R}}$ given by the conjugation by $\operatorname{ad}\left(h_{W}(i)\right)$.

Let $K$ be a maximal compact subgroup of $\mathrm{Hg}(\mathcal{X})_{\mathbb{R}}$. Since all maximal compact subgroups of a reductive group are conjugate, we assume without loss of generality that $K$ is the subgroup fixed by the Cartan involution obtained from conjugation by $h_{W}(i)$. Let $C((\operatorname{ad} \circ h)(i))$ denote the centralizer of $(\operatorname{ad} \circ h)(i)$ in $\mathrm{Hg}^{\text {ad }}(\mathcal{X})$.

LEMMA 1.12.

$$
C((\operatorname{ad} \circ h)(i))=\operatorname{ad}(K)=\operatorname{ad}\left(K \cap \operatorname{Hg}^{\operatorname{der}}(\mathcal{X})_{\mathbb{R}}\right) .
$$

Proof. One has clearly

$$
C((\operatorname{ad} \circ h)(i)) \supseteq \operatorname{ad}(K) \supseteq \operatorname{ad}\left(K \cap \operatorname{Hg}^{\operatorname{der}}(\mathcal{X})_{\mathbb{R}}\right) .
$$

Thus it remains to prove

$$
C((\operatorname{ad} \circ h)(i)) \subseteq \operatorname{ad}\left(K \cap \operatorname{Hg}^{\operatorname{der}}(\mathcal{X})_{\mathbb{R}}\right) .
$$

Since $\mathrm{Hg}^{\text {ad }}(\mathcal{X})_{\mathbb{R}}$ and $\mathrm{Hg}^{\text {der }}(\mathcal{X})_{\mathbb{R}}$ are isogenous, we have a correspondence between their maximal compact subgroups. The maximal compact subgroups $K_{G}$ of real algebraic reductive groups $G$ are the subgroups of $G$ satisfying

$$
K_{G}=\{g \in G \quad \mid \theta(g)=g\}
$$

for some Cartan involution $\theta$ (follows from [12], I. Corollary 4.3 and Corollary 4.5). Recall that the conjugation by $h(i)$ yields a Cartan involution on $\operatorname{Hg}^{\text {der }}(\mathcal{X})_{\mathbb{R}}$ and the conjugation by $(\operatorname{ad} \circ h)(i)$ yields a Cartan involution on $\operatorname{Hg}^{\text {ad }}(\mathcal{X})_{\mathbb{R}}$. Thus one concludes that the centralizer of $(\operatorname{ad} \circ h)(i)$ is given by $\operatorname{ad}\left(K \cap \operatorname{Hg}^{\text {der }}(\mathcal{X})_{\mathbb{R}}\right)$.

2. Computation of the adjoint Hodge group. In this section we prove the following theorem:

TheOREM 2.1. The group $\mathrm{Hg}^{\mathrm{ad}}(\mathcal{X})_{\mathbb{R}}$ is isomorphic to $\mathrm{PU}(1,1)$ or $\mathrm{Sp}_{\mathbb{R}}^{\mathrm{ad}}(4)$.

For the proof of Theorem 2.1 we need to understand $K$ first:

Lemma 2.2. The group $K^{0}$ is a torus or $K=C\left(h_{W}(i)\right)$. 
Proof. Since $K^{0}$ is compact, $K^{0}$ is reductive (see 1.3). One has without loss of generality

$$
K \subseteq C\left(h_{W}(i)\right) \cong \mathrm{U}(2) .
$$

If $K^{0}$ is a torus, we are done. Otherwise $K^{0}$ has a nontrivial semisimple subgroup

$$
K^{\text {der }} \subseteq C^{\text {der }}\left(h_{W}(i)\right) \cong \mathrm{SU}(2)
$$

(see 1.1). Since $\mathrm{SU}(2)$ does not contain any simple proper subgroup, $K^{\text {der }}=$ $C^{\operatorname{der}}\left(h_{W}(i)\right)$. From the facts that $h\left(S^{1}\right)$ is not contained in $C^{\operatorname{der}}\left(h_{W}(i)\right)$, but contained in $\operatorname{Hg}(\mathcal{X})_{\mathbb{R}}$ and commutes with $h_{W}(i)=h(-i)$, we conclude $K=C\left(h_{W}(i)\right)$ in this case.

Lemma 2.3. The centralizer of $C^{\operatorname{der}}\left(h_{W}(i)\right)$ in $\operatorname{Sp}\left(H^{3}(X, \mathbb{R}), Q\right)$ is given by the center $Z\left(C\left(h_{W}(i)\right)\right)$ of $C\left(h_{W}(i)\right)$.

Proof. Recall the description of $C^{\mathrm{der}}\left(h_{W}(i)\right) \cong \mathrm{SU}(2)$ in Proposition 1.7 and the description of $\mathrm{SU}(2)$ in Remark 1.8. Thus $N \in C^{\operatorname{der}}\left(h_{W}(i)\right)(\mathbb{R})$ is given by

$$
N=\left(\begin{array}{cccc}
a & b & 0 & 0 \\
-\bar{b} & \bar{a} & 0 & 0 \\
0 & 0 & a & -b \\
0 & 0 & \bar{b} & \bar{a}
\end{array}\right) \text { with }|a|^{2}+|b|^{2}=1
$$

with respect to the basis $\left\{v_{3,0}, v_{1,2}, v_{2,1}, v_{0,3}\right\}$. Now let

$$
M=\left(\begin{array}{cc}
A & B \\
C & D
\end{array}\right) \in \operatorname{Sp}\left(H^{3}(X, \mathbb{R}), Q\right)(\mathbb{R})
$$

commute with each $N \in C^{\operatorname{der}}\left(h_{W}(i)\right)(\mathbb{R})$ for some suitable $A, B, C, D \in \mathrm{GL}_{2}(\mathbb{C})$. Thus $M$ commutes with $\operatorname{diag}(i,-i, i,-i)$ and one computes that $A, B, C, D$ are diagonal matrices. Moreover one has that $M$ has to commute with

$$
N=\left(\begin{array}{cccc}
0 & 1 & 0 & 0 \\
-1 & 0 & 0 & 0 \\
0 & 0 & 0 & -1 \\
0 & 0 & 1 & 0
\end{array}\right)
$$

From this fact and the assumptions that $M$ is a real matrix and commutes with each element of $C^{\mathrm{der}}\left(h_{W}(i)\right)(\mathbb{R})$, one concludes

$$
M=\left(\begin{array}{cccc}
z & 0 & \bar{y} & 0 \\
0 & z & 0 & -\bar{y} \\
-y & 0 & \bar{z} & 0 \\
0 & y & 0 & \bar{z}
\end{array}\right)
$$

Moreover one computes that

$$
\begin{aligned}
M^{t} Q M & =\left(\begin{array}{cccc}
z & 0 & -y & 0 \\
0 & z & 0 & y \\
\bar{y} & 0 & \bar{z} & 0 \\
0 & -\bar{y} & 0 & \bar{z}
\end{array}\right)\left(\begin{array}{cccc}
0 & 0 & 0 & -i \\
0 & 0 & -i & 0 \\
0 & i & 0 & 0 \\
i & 0 & 0 & 0
\end{array}\right)\left(\begin{array}{cccc}
z & 0 & \bar{y} & 0 \\
0 & z & 0 & -\bar{y} \\
-y & 0 & \bar{z} & 0 \\
0 & y & 0 & \bar{z}
\end{array}\right) \\
& =\left(\begin{array}{cccc}
0 & -2 i y z & 0 & i|y|^{2}-i|z|^{2} \\
2 i y z & 0 & i|y|^{2}-i|z|^{2} & 0 \\
0 & -i|y|^{2}+i|z|^{2} & 0 & -2 i \bar{y} \bar{z} \\
-i|y|^{2}+i|z|^{2} & 0 & 2 i \bar{y} \bar{z} & 0
\end{array}\right) .
\end{aligned}
$$


Hence $M \in \operatorname{Sp}\left(H^{3}(X, \mathbb{R}), Q\right)$, only if $y=0$ and $|z|=1$. Thus $M \in Z\left(C\left(h_{W}(i)\right)\right)$.

LEMMA 2.4. $\operatorname{Hg}(\mathcal{X})_{\mathbb{R}}$ cannot be compact.

Proof. Assume that $\mathrm{Hg}(\mathcal{X})_{\mathbb{R}}$ would be compact. Thus one concludes that $\operatorname{Hg}(\mathcal{X})_{\mathbb{R}}=K$ is a torus or $\operatorname{Hg}(\mathcal{X})_{\mathbb{R}}=C\left(h_{W}(i)\right)$ (see Lemma 2.2). In the first case one concludes $\operatorname{Hg}(\mathcal{X})_{\mathbb{R}} \subseteq C\left(h\left(S^{1}\right)\right)$, which contains only 4 complex structures (see Remark 1.5). In the second case the Cartan involution obtained from conjugation by $h_{\mathcal{X}_{b}}(i) \in C\left(h_{W}(i)\right)$ fixes each element of the compact group $\operatorname{Hg}(\mathcal{X})(\mathbb{R})=C\left(h_{W}(i)\right)(\mathbb{R})$ for each $b \in B$. Hence each $h_{\mathcal{X}_{b}}(i)$ has to be contained in the center of $C\left(h_{W}(i)\right)$. Note that $Z\left(C\left(h_{W}(i)\right)\right)$ has only the two complex structures $\pm h_{W}(i)$. Thus in any case $h(i)=h_{\mathcal{X}_{b}}(i)$ for each $b \in B$, since the $V H S$ is continuous and for each $b \in B$ one obtains

$$
H^{3,0}\left(\mathcal{X}_{b}\right) \subset \operatorname{Eig}\left(h_{\mathcal{X}_{b}}(i),-i\right)=\operatorname{Eig}\left(h_{X}(i),-i\right)=\operatorname{Span}\left(v_{3,0}, v_{1,2}\right) .
$$

But this contradicts the fact that $\omega(0)$ and $\nabla_{\frac{\partial}{\partial b}} \omega(0)$ generate $F^{2}(X)$, where $\omega$ denotes a generic section of the $F^{3}$-bundle in the $V H S$ (see [2]).

Now we change for a moment to the language of semisimple adjoint Lie groups. Connected semisimple adjoint Lie groups are direct products of their normal simple subgroups (see [10], Lemma 1.3.8). The group $\mathrm{Hg}^{\text {ad }}(\mathcal{X})(\mathbb{R})^{+}$is an example of a connected semisimple adjoint Lie group.

Proposition 2.5. There does not exist any nontrivial direct factor $F$ of $\mathrm{Hg}^{\mathrm{ad}}(\mathcal{X})(\mathbb{R})^{+}$such that

$$
Z(K)(\mathbb{R})^{+} \subset \operatorname{ker}\left(p r_{F} \circ \operatorname{ad}\right)
$$

Proof. Assume that $F$ is a direct factor of $\mathrm{Hg}^{\text {ad }}(\mathcal{X})(\mathbb{R})^{+}$with

$$
Z(K)(\mathbb{R})^{+} \subset \operatorname{ker}\left(p r_{F} \circ \operatorname{ad}\right) .
$$

We show that $F$ is trivial. Since $\operatorname{Hg}(\mathcal{X})_{\mathbb{R}}$ cannot be compact (see Lemma 2.4), the maximal compact subgroup $K$ associated to the Cartan involution obtained from conjugation by $h(i)$ is a proper subgroup. Thus $h(i)$ is not contained in the center of $\operatorname{Hg}(\mathcal{X})_{\mathbb{R}}$. Since $h\left(S^{1}\right)(\mathbb{R})$ is connected, $h\left(S^{1}\right)(\mathbb{R}) \subset Z(K)(\mathbb{R})^{+}$, which implies that $h(i) \in Z(K)(\mathbb{R})^{+}$. Thus from our assumption we conclude that $F$ is contained in the maximal compact subgroup associated to the Cartan involution obtained from conjugation by $(\operatorname{ad} \circ h)(i)$. Consider the projection map $\operatorname{pr}_{F}: \mathrm{Hg}^{\text {ad }}(\mathcal{X})(\mathbb{R})^{+} \rightarrow F$. Since

$$
(\operatorname{ad} \circ h)(i) \in G:=\operatorname{ker}\left(p r_{F}\right) \subset \operatorname{Hg}^{\operatorname{ad}}(\mathcal{X})(\mathbb{R})^{+},
$$

one concludes that $G$ is non-trivial semisimple adjoint. Note that

$$
\operatorname{ker}\left(p r_{G}\right)=F \text { and } \operatorname{Hg}^{\text {ad }}(\mathcal{X})(\mathbb{R})^{+}=F \times G
$$

since connected semisimple adjoint Lie groups are direct products of thier normal simple subgroups (see [10], Lemma 1.3.8). Let

$$
F^{\prime}=\operatorname{ker}\left(\left.p r_{G} \circ \operatorname{ad}\right|_{\operatorname{Hg}^{\text {der }}(\mathcal{X})(\mathbb{R})}\right)^{+} \text {and } G^{\prime}=\operatorname{ker}\left(\left.p r_{F} \circ \operatorname{ad}\right|_{\operatorname{Hg}^{\text {der }}(\mathcal{X})(\mathbb{R})}\right)^{+} \text {. }
$$


Since $\operatorname{Hg}^{\text {ad }}(\mathcal{X})_{\mathbb{R}}$ and $\operatorname{Hg}^{\text {der }}(\mathcal{X})_{\mathbb{R}}$ are isogenous, one concludes that $F^{\prime}$ and $G^{\prime}$ commute. Since $F^{\prime}$ is a semisimple group fixed by the Cartan involution obtained from conjugation by $h(i)$ and $C^{\text {der }}(h(i))(\mathbb{R}) \cong \mathrm{SU}(2)(\mathbb{R})$ contains no semisimple proper subgroup, one concludes

$$
F^{\prime}=C^{\text {der }}(h(i))(\mathbb{R}) \text { or } F^{\prime}=\{e\} .
$$

Only the torus $Z(C(h(i)))$ commutes with $C^{\text {der }}(h(i))$ (see Lemma 2.3). Thus from the fact that $G^{\prime}$ is nontrivial semisimple and commutes with $F^{\prime}$, we conclude $F^{\prime}=\{e\}$. Thus $F$ is trivial.

The connected semisimple adjoint Lie group $\mathrm{Hg}^{\text {ad }}(\mathcal{X})(\mathbb{R})^{+}$is a direct product of connected simple adjoint subgroups. Let $F$ be one of these direct factors. The maximal compact subgroup of $\mathrm{Hg}^{\text {ad }}(\mathcal{X})(\mathbb{R})^{+}$is given by

$$
\operatorname{ad}(K(\mathbb{R})) \cap \operatorname{Hg}^{\text {ad }}(\mathcal{X})(\mathbb{R})^{+}
$$

(follows from Lemma 1.12). Thus for the maximal compact subgroup $K_{F}$ of $F$ one concludes that $K_{F}^{+}=\left(p r_{F} \circ \mathrm{ad}\right)\left(K(\mathbb{R})^{+}\right)$. Due to the fact that $Z(K)(\mathbb{R})^{+}$is not contained in $\operatorname{ker}\left(p r_{F} \circ \mathrm{ad}\right)$ and not discrete as one concludes from Lemma 2.2, the maximal compact subgroup $K_{F}$ has a nondiscrete center. Since $F$ has a trivial center, $K_{F} \neq F$ and one concludes:

Corollary 2.6. The connected adjoint Lie group $\mathrm{Hg}^{\mathrm{ad}}(\mathcal{X})(\mathbb{R})^{+}$is a direct product of noncompact simple adjoint subgroups, whose maximal compact subgroups have nondiscrete centers.

Note that each Hermitian symmetric domain is a direct product of irreducible Hermitian symmetric domains (for the definition and more details about Hermitian symmetric domains see [6]). If $G$ is a connected simple adjoint noncompact Lie group and $K_{G}$ is a maximal compact subgroup of $G$ with nondiscrete center, the quotient $G / K_{G}$ has the structure of a uniquely determined irreducible Hermitian symmetric domain ([6], XIII. Theorem 6.1,). Hence one concludes from Corollary 2.6:

Proposition 2.7. The quotient

$$
D=\mathrm{Hg}^{\mathrm{ad}}(\mathcal{X})(\mathbb{R})^{+} / \operatorname{ad}(K(\mathbb{R})) \cap \mathrm{Hg}^{\mathrm{ad}}(\mathcal{X})(\mathbb{R})^{+}
$$

has the structure of an Hermitian symmetric domain.

Since $\operatorname{Hg}(\mathcal{X})_{\mathbb{R}} \subset \operatorname{Sp}\left(H^{3}(X, \mathbb{R}), Q\right)$, the associated Hermitian symmetric domain of $\operatorname{Sp}\left(H^{3}(X, \mathbb{Q}), Q\right)(\mathbb{R})$ is $\mathfrak{h}_{2}$ and $\operatorname{dim}_{\mathbb{C}} \mathfrak{h}_{2}=3$, the Hermitian symmetric domain $D$ has dimension 1,2 or 3 . By using these conditions, we obtain some candidates for $\mathrm{Hg}^{\text {ad }}(\mathcal{X})(\mathbb{R})^{+}$. Since these candidates are the Lie groups of real valued points of $\mathbb{R}$ algebraic semisimple adjoint groups, we obtain not only connected Lie groups, but $\mathbb{R}$-algebraic groups in our cases by using Lemma 1.2. Moreover we will exclude all of these candidates except of the candidates stated in Theorem 2.1.

LEMMA 2.8. If D has dimension one, we obtain

$$
\operatorname{Hg}^{\mathrm{ad}}(\mathcal{X})_{\mathbb{R}} \cong \mathrm{PU}(1,1)
$$

Proof. Assume that $D$ has dimension one. By consulting the list of irreducible Hermitian symmetric domains $([6], \mathbf{X}$, Table $\mathbf{V})$, one concludes $D=\mathbb{B}_{1}$. Thus from the fact that there are no direct compact factors (see Corollary 2.6) one concludes

$$
\mathrm{Hg}^{\mathrm{ad}}(\mathcal{X})_{\mathbb{R}} \cong \mathrm{PU}(1,1) \text {. }
$$


LEMmA 2.9. If D has dimension two, we obtain

$$
\operatorname{Hg}^{\text {ad }}(\mathcal{X})_{\mathbb{R}} \cong \mathrm{PU}(1,2), \text { or } \operatorname{Hg}^{\text {ad }}(\mathcal{X})_{\mathbb{R}} \cong \mathrm{PU}(1,1) \times \mathrm{PU}(1,1) \text {. }
$$

Proof. By consulting the list of irreducible Hermitian symmetric domains ([6], X, Table $\mathbf{V}$ ), the only possible Hermitian symmetric domains of dimension two are up to isomorphisms given by $\mathbb{B}_{1} \times \mathbb{B}_{1}$ and $\mathbb{B}_{2}$. Thus we obtain the stated result.

Lemma 2.10. One obtains $\operatorname{Hg}(\mathcal{X})=\operatorname{Sp}\left(H^{3}(X, \mathbb{Q}), Q\right)$, if $D$ has the dimension 3.

Proof. We show that $\mathfrak{h}_{2}$ contains no bounded symmetric domain of dimension 3 except of itself. In order to do this we check the list of Hermitian Symmetric Domains (compare [6], $\mathbf{X}$, Table $\mathbf{V}$ ). The domain $D$ cannot be the direct product of 3 copies of $\mathbb{B}_{1}$, since in this case the centralizer of $\left(\operatorname{ad} \circ h_{X}\right)(i)$ would be a torus of dimension 3 . But the centralizer of $h_{X}(i)$ is isomorphic to $\mathrm{U}(2)$, which contains a maximal torus of dimension 2. Since each point $p \in \mathbb{B}_{1} \times \mathbb{B}_{2}$ has a centralizer $S^{1} \times \mathrm{U}(2)$ of dimension 5 and $C(h(i)) \cong \mathrm{U}(2)$ has dimension 4 , one concludes that $D$ cannot be isomorphic to $\mathbb{B}_{1} \times \mathbb{B}_{2}$. In the case of $\mathbb{B}_{3}$ the stabilizer is $\mathrm{U}(3)$ and hence it is to large. The same holds true in the case of $\mathrm{SO}^{*}(6) / \mathrm{U}(3)$. Moreover the associated bounded symmetric domain of $\mathrm{SO}(2,3)^{+}(\mathbb{R})$ is isomorphic to $\mathfrak{h}_{2}$. Thus we obtain the stated result.

By the previous lemmas, the following adjoint semisimple groups are possible candidates for $\mathrm{Hg}^{\text {ad }}(\mathcal{X})_{\mathbb{R}}$ :

$$
\mathrm{PU}(1,1), \quad \mathrm{PU}(1,1) \times \mathrm{PU}(1,1), \quad \mathrm{PU}(1,2), \quad \mathrm{Sp}_{\mathbb{R}}^{\mathrm{ad}}(4) .
$$

Now we exclude $\mathrm{PU}(1,2)$ and $\mathrm{PU}(1,1) \times \mathrm{PU}(1,1)$.

Proposition 2.11. The group $\mathrm{Hg}^{\text {ad }}(\mathcal{X})_{\mathbb{R}}$ cannot be isomorphic to $\mathrm{PU}(1,2)$.

Proof. Assume that $\mathrm{Hg}^{\text {ad }}(\mathcal{X})_{\mathbb{R}}$ would be isomorphic to $\mathrm{PU}(1,2)$. In this case the centralizer $C((\operatorname{ad} \circ h)(i)) \subset \mathrm{Hg}^{\text {ad }}(\mathcal{X})_{\mathbb{R}}$ of the complex structure $($ adoh $)(i)$ is isomorphic to $\mathrm{U}(2)$. Hence $C((\operatorname{ad} \circ h)(i))$ has dimension 4 . One has that $C((\operatorname{ad} \circ h)(i))$ is isogenous to $C(h(i)) \cap \operatorname{Hg}^{\text {der }}(\mathcal{X})_{\mathbb{R}}$. Since $C(h(i))$ has already dimension 4 and $h\left(S^{1}\right) \subset C(h(i))$, one concludes

$$
C(h(i)) \subset \operatorname{Hg}^{\operatorname{der}}(\mathcal{X})_{\mathbb{R}} \text { and } \operatorname{Hg}^{\operatorname{der}}(\mathcal{X})_{\mathbb{R}}=\operatorname{Hg}(\mathcal{X})_{\mathbb{R}}
$$

Note that

$$
C^{\mathrm{der}}(h(i)) \cong \mathrm{SU}(2)
$$

Moreover ad yields a homomorphism

$$
g:=\left.\operatorname{ad}\right|_{C^{\operatorname{der}}(h(i))}: C^{\operatorname{der}}(h(i)) \rightarrow C(\operatorname{ad} \circ h(i)),
$$

whose kernel consists of $\{ \pm \mathrm{id}\}$. Since

$$
C^{\text {der }}(h(i)) /\{ \pm \mathrm{id}\} \cong \mathrm{PU}(2)
$$

is semisimple, one has

$$
\left(g\left(C^{\text {der }}(h(i))\right)\right)^{\text {der }}=g\left(C^{\text {der }}(h(i))\right) .
$$


Hence

$$
g\left(C^{\text {der }}(h(i))\right) \subset C^{\text {der }}(\operatorname{ad} \circ h(i)) \cong \mathrm{SU}(2)
$$

Recall that

$$
\mathrm{SU}(2)(\mathbb{R})=\left\{M(\alpha, \beta)=\left(\begin{array}{cc}
\alpha & \beta \\
-\bar{\beta} & \bar{\alpha}
\end{array}\right):|\alpha|^{2}+|\beta|^{2}=1\right\} .
$$

Each matrix $M(\alpha, \beta) \in \mathrm{SU}(2)(\mathbb{R})$ with $\alpha \in i \mathbb{R}$ has the characteristic polynomial

$$
x^{2}+1=(x-i)(x+i),
$$

which implies that $M(\alpha, \beta)$ is a complex structure. Therefore $C^{\operatorname{der}}(h(i))(\mathbb{R}) \cong$ $\mathrm{SU}(2)(\mathbb{R})$ contains infinitely many complex structures. Since $\operatorname{ker}(g)=\{ \pm \mathrm{id}\}$, all these complex structures are mapped to infinitely many elements of order 2 in $C^{\text {der }}(\operatorname{ado} h(i))$. Since each $2 \times 2$ matrix $M$ of order 2 has a minimal polynomial dividing the polynomial $x^{2}-1$, the matrix $M$ is either given by $\operatorname{diag}(-1,-1)$ or one has an eigenspace with respect to 1 and one eigenspace with respect to -1 . In the second case $\operatorname{det}(M)=-1$. Thus $\operatorname{diag}(-1,-1)$ is the only element of order 2 in $\mathrm{SU}(2)(\mathbb{R})$. On the other hand there are infinitely many complex structures in $C^{\text {der }}(h(i))(\mathbb{R})$, which are mapped by $g$ to infinitely many elements of order 2 in $C((\operatorname{ad} \circ h)(i))(\mathbb{R}) \cong \mathrm{SU}(2)(\mathbb{R})$. Thus we have a contradiction.

Let $H$ denote the centralizer of $h_{G}(i) h_{W}(i)$ in $\operatorname{Sp}\left(H^{3}(X, \mathbb{R}), Q\right)$. Note that

$$
h_{G}(i) h_{W}(i)=\operatorname{diag}(-1,-1,1,1)
$$

with respect to the basis $\left\{v_{3,0}, v_{0,3}, v_{2,1}, v_{1,2}\right\}$. Thus $H(\mathbb{R})$ is given by the matrices

$$
M_{1}=\left(\begin{array}{cccc}
a & b & 0 & 0 \\
\bar{b} & \bar{a} & 0 & 0 \\
0 & 0 & c & d \\
0 & 0 & \bar{d} & \bar{c}
\end{array}\right) \quad \text { with }\left(\begin{array}{cc}
a & b \\
\bar{b} & \bar{a}
\end{array}\right),\left(\begin{array}{cc}
c & d \\
\bar{d} & \bar{c}
\end{array}\right) \in \operatorname{SU}(1,1)(\mathbb{R})
$$

with respect to the basis $\left\{v_{3,0}, v_{0,3}, v_{2,1}, v_{1,2}\right\}$. One can easily verify this fact by explicit computations using the description of the symplectic form $Q$ in (1). The group $H$ will play an important role due to the following lemma:

Lemma 2.12. The group $\operatorname{Hg}(\mathcal{X})_{\mathbb{R}}$ cannot be a subgroup of $H$.

Proof. Assume that $\operatorname{Hg}(\mathcal{X})_{\mathbb{R}}$ would be a subgroup of $H$. Since for each $b \in B$ the conjugation by $h_{W}(i)_{b}$ yields a Cartan involution of $\operatorname{Sp}\left(H^{3}(X, \mathbb{R}), Q\right)$, which can be restricted to an involution of $H$ in this case, the conjugation by $h_{W}(i)_{b}$ yields a Cartan involution of $H$ (compare [12], I. Theorem 4.2). Due to the fact $H \cong \operatorname{SU}(1,1) \times$ $\mathrm{SU}(1,1)$, the corresponding maximal compact subgroup is a torus of dimension 2 containing $h_{b}\left(S^{1}\right)$. By Remark 1.5, the centralizer $C\left(h_{b}\left(S^{1}\right)\right)$ is already a torus of dimension 2. Hence

$$
h_{G}(i)_{b} \in C\left(h_{b}\left(S^{1}\right)\right) \subset H .
$$

Thus from the description of $H$ in (3) and the fact that $h_{G}(i)_{b}, h_{W}(i)_{b} \in H$ are real complex structures, one concludes that

$$
\operatorname{Eig}\left(h_{G}(i)_{b}, i\right)=\operatorname{Span}\left(v_{1}, v_{3}\right), \quad \operatorname{Eig}\left(h_{W}(i)_{b}, i\right)=\operatorname{Span}\left(v_{2}, v_{4}\right)
$$


with

$$
v_{1}, v_{2} \in \operatorname{Span}\left(v_{3,0}, v_{0,3}\right), \quad v_{3}, v_{4} \in \operatorname{Span}\left(v_{2,1}, v_{1,2}\right) .
$$

For each $b \in B$ one has the onedimensional vector space

$$
H^{3,0}\left(\mathcal{X}_{b}\right)=\operatorname{Eig}\left(h_{G}(i)_{b}, i\right) \cap \operatorname{Eig}\left(h_{W}(i)_{b}, i\right) .
$$

Hence $\left\{v_{1}, \ldots, v_{4}\right\}$ is not linearly independent and one concludes from the description of $H$ in $(4)$ that $H^{3,0}\left(\mathcal{X}_{b}\right)$ is either contained in $\operatorname{Span}\left(v_{3,0}, v_{0,3}\right)$ or contained in $\operatorname{Span}\left(v_{2,1}, v_{1,2}\right){ }^{3}$ Since the period map is continuous, one has for each $b \in B$

$$
H^{3,0}\left(\mathcal{X}_{b}\right) \subset \operatorname{Span}\left(v_{3,0}, v_{0,3}\right) .
$$

This contradicts the fact that $\omega(0)$ and $\nabla_{\frac{\partial}{\partial b}} \omega(0)$ generate $F^{2}(X)$, where $\omega$ denotes a generic section of the $F^{3}$-bundle in the $V H S$ (see [2]). Thus $\operatorname{Hg}(\mathcal{X})_{\mathbb{R}}$ cannot be a subgroup of $H$.

Proposition 2.13. One cannot have

$$
\operatorname{Hg}^{\mathrm{ad}}(\mathcal{X})_{\mathbb{R}} \cong \mathrm{PU}(1,1) \times \mathrm{PU}(1,1)
$$

Proof. Assume that $\mathrm{Hg}^{\text {ad }}(\mathcal{X})_{\mathbb{R}} \cong \mathrm{PU}(1,1) \times \mathrm{PU}(1,1)$. Without loss of generality the only possible Cartan involution of $\mathrm{PU}(1,1) \times \mathrm{PU}(1,1)$ is given by the conjugation by

$$
([\operatorname{diag}(i,-i)],[\operatorname{diag}(i,-i)]) \in \mathrm{PU}(1,1) \times \mathrm{PU}(1,1) .
$$

Moreover in $\operatorname{Hg}^{\text {ad }}(\mathcal{X})_{\mathbb{R}} \cong \mathrm{PU}(1,1) \times \mathrm{PU}(1,1)$ the maximal compact subgroup of elements fixed by the Cartan involution is given by a torus of dimension 2 . Thus there is a torus $T \subset \mathrm{Hg}^{\text {der }}(\mathcal{X})_{\mathbb{R}}$ of dimension two, whose elements are fixed by the Cartan involution. Assume without loss of generality that the Cartan involution of $\operatorname{Hg}^{\text {der }}(\mathcal{X})_{\mathbb{R}}$ is obtained from conjugation by $h(i)$. Thus $T$ is a maximal torus of $C(h(i)) \cong \mathrm{U}(2)$, since $T$ has dimension 2. Therefore the center of $\operatorname{Hg}(\mathcal{X})_{\mathbb{R}}$ is discrete and one concludes from 1.1 that

$$
\operatorname{Hg}^{\text {der }}(\mathcal{X})_{\mathbb{R}}=\operatorname{Hg}(\mathcal{X})_{\mathbb{R}}
$$

From the fact that each element of $h\left(S^{1}\right)$ commutes with $h(i)$, one concludes $h\left(S^{1}\right) \subset$ $T$. Since $T$ is a torus of dimension 2 containing $h\left(S^{1}\right)$, one concludes from Remark 1.5 that $T=C\left(h\left(S^{1}\right)\right)$. Thus $h_{G}(i) \in T$ and $h_{G}\left(S^{1}\right) \subset T$. Note that $h_{G}(i)$ cannot be contained in the center of $\operatorname{Hg}(\mathcal{X})_{\mathbb{R}}$, since $h_{G}(i) \in Z\left(\operatorname{Hg}(\mathcal{X})_{\mathbb{R}}\right)$ would imply that $h_{G}\left(S^{1}\right) \subset Z\left(\operatorname{Hg}(\mathcal{X})_{\mathbb{R}}\right)$ as one can easily conclude from the fact that

$$
h_{G}\left(S^{1}\right)(\mathbb{R})=\left\{a \cdot \mathrm{id}+b \cdot h_{G}(i) \mid a^{2}+b^{2}=1\right\} .
$$

This contradicts our conclusion that $Z\left(\operatorname{Hg}(\mathcal{X})_{\mathbb{R}}\right)$ is discrete. Since $h_{G}(i)$ has order 4 and

$$
h_{G}(i)^{2}=-\mathrm{id} \in \operatorname{ker}(\operatorname{ad}),
$$

\footnotetext{
${ }^{3}$ This is only an exercise in linear algebra.
} 
one concludes that $\operatorname{ad}\left(h_{G}(i)\right)$ yields an element of order two in $\operatorname{ad}(T)$. Note that $\operatorname{ad}(T)$ has only the three elements

$([\operatorname{diag}(i,-i)],[\operatorname{diag}(1,1)]), \quad([\operatorname{diag}(i,-i)],[\operatorname{diag}(i,-i)])$ and $([\operatorname{diag}(1,1)],[\operatorname{diag}(i,-i)])$

of order 2. Thus we have two cases: In the first case $\operatorname{ad}\left(h_{G}(i)\right)$ is without loss of generality given by

$$
([\operatorname{diag}(i,-i)],[\operatorname{diag}(1,1)]) .
$$

Let $\operatorname{pr}_{i}(i=1,2)$ denote the projection of $\mathrm{Hg}^{\text {ad }}(\mathcal{X})_{\mathbb{R}} \cong \mathrm{PU}(1,1) \times \mathrm{PU}(1,1)$ to the respective copy of $\mathrm{PU}(1,1)$. One has that $\operatorname{Hg}(\mathcal{X})_{\mathbb{R}}$ contains $\operatorname{ker}\left(p r_{1} \circ \text { ad }\right)^{0}$ and $\operatorname{ker}\left(p r_{2} \circ\right.$ ad $)^{0}$. Since the groups $\mathrm{Hg}^{\text {ad }}(\mathcal{X})_{\mathbb{R}}$ and $\operatorname{Hg}^{\text {der }}(\mathcal{X})_{\mathbb{R}}=\mathrm{Hg}(\mathcal{X})_{\mathbb{R}}$ are isogenous, $\operatorname{ker}\left(p r_{1}\right.$ 。 ad $)^{0}$ and $\operatorname{ker}\left(p r_{2} \circ \mathrm{ad}\right)^{0}$ are also isogenous to $\mathrm{PU}(1,1)$ and commute also. Moreover since $\mathrm{Hg}^{\text {ad }}(\mathcal{X})_{\mathbb{R}}$ and $\operatorname{Hg}(\mathcal{X})_{\mathbb{R}}$ are isogenous, $\left(\operatorname{Hg}(\mathcal{X})_{\mathbb{R}} \cap C\left(h_{G}(i)\right)\right)^{0}$ is also isogenous to $C\left(\left(\operatorname{ad} \circ h_{G}\right)(i)\right)$. Since $\operatorname{ker}\left(p r_{1}\right)$ commutes with $\operatorname{ad}\left(h_{G}(i)\right)$, one concludes that $\operatorname{ker}\left(p r_{1} \circ \mathrm{ad}\right)^{0}$ is a nontrivial simple subgroup of $C\left(h_{G}(i)\right)$. Since the only nontrivial simple subgroup of $C\left(h_{G}(i)\right)$ is $C^{\operatorname{der}}\left(h_{G}(i)\right)$, one gets

$$
\operatorname{ker}\left(p r_{1} \circ \mathrm{ad}\right)^{0}=C^{\mathrm{der}}\left(h_{G}(i)\right) .
$$

By analogue arguments, one concludes

$$
\operatorname{ker}\left(p r_{2} \circ \mathrm{ad}\right)^{0} \subset H:=C\left(h_{G}(i) h_{W}(i)\right) .
$$

We obtain the desired contradiction by showing that $\operatorname{ker}\left(p r_{1} \circ \text { ad }\right)^{0}$ and $\operatorname{ker}\left(p r_{2} \circ \text { ad }\right)^{0}$ cannot commute here. One has that $C^{\operatorname{der}}\left(h_{G}(i)\right)(\mathbb{R})$ is given by matrices of the form

$$
M_{2}=\left(\begin{array}{cccc}
\alpha & 0 & \beta & 0 \\
0 & \bar{\alpha} & 0 & \bar{\beta} \\
\bar{\beta} & 0 & \bar{\alpha} & 0 \\
0 & \beta & 0 & \alpha
\end{array}\right) \text { with }|\alpha|^{2}-|\beta|^{2}=1
$$

with respect to the basis

$$
\left\{v_{3,0}, v_{0,3}, v_{2,1}, v_{1,2}\right\}
$$

as the reader can easily verify by the description of $C\left(h_{G}(i)\right)(\mathbb{R}) \cong \mathrm{U}(1,1)$ in Proposition 1.6 and the description of $\mathrm{SU}(1,1)$ in Remark 1.8. Moreover by explicit computations using $(3)$, one checks that in $H(\mathbb{R})$ only the diagonal matrices of the kind $\operatorname{diag}(\xi, \bar{\xi}, \xi, \bar{\xi})$ commute with each element of $C^{\operatorname{der}}\left(h_{G}(i)\right)(\mathbb{R})$. This contradicts our previous conclusion that $H$ contains a subgroup isogenous to $\mathrm{PU}(1,1)$, which commutes with $C^{\operatorname{der}}\left(h_{G}(i)\right)(\mathbb{R})$. Hence the first case cannot hold true.

In the second case $\operatorname{ad}\left(h_{G}(i)\right)$ is given by

$$
([\operatorname{diag}(i,-i)],[\operatorname{diag}(i,-i)]) \in \mathrm{PU}(1,1) \times \mathrm{PU}(1,1) .
$$

This implies that $\mathrm{Hg}^{\mathrm{der}}(\mathcal{X})=\operatorname{Hg}(\mathcal{X})_{\mathbb{R}}$ is contained in the subgroup of $\operatorname{Sp}\left(H^{3}(X, \mathbb{R}), Q\right)$ on which both involutions obtained from conjugation by $h_{W}(i)$ and $h_{G}(i)$ coincide. One has that

$$
h_{W}(i)=\operatorname{diag}(i,-i,-i, i) \text { and } h_{G}(i)=\operatorname{diag}(i,-i, i,-i)
$$


with respect to the basis

$$
\left\{v_{3,0}, v_{0,3}, v_{2,1}, v_{1,2}\right\} \text {. }
$$

Thus $H$ is the subgroup of $\operatorname{Sp}\left(H^{3}(X, \mathbb{R})\right)$ on which both involutions obtained from conjugation by $h_{W}(i)$ and $h_{G}(i)$ coincide as one can easily compute by using the description of $H$ in (3). But by Lemma 2.12, the group $H$ cannot contain $\operatorname{Hg}(\mathcal{X})_{\mathbb{R}}$. Thus the second case cannot occur.

3. The case of a onedimensional period domain. In this section we will assume that the period domain $D$ has dimension 1 unless stated otherwise. In the previous section we saw that $\operatorname{Hg}^{\text {ad }}(\mathcal{X})_{\mathbb{R}} \cong \mathrm{PU}(1,1)$, if $D=1$. Since

$$
\operatorname{Hg}(\mathcal{X})=\left(\operatorname{SL}\left(H^{3}(X, \mathbb{Q})\right) \cap \operatorname{MT}(\mathcal{X})\right)^{0}
$$

(follows from [10], Lemma 1.3.17), one concludes

$$
\operatorname{Hg}^{\mathrm{ad}}(\mathcal{X})=\operatorname{MT}^{\mathrm{ad}}(\mathcal{X})
$$

Recall the definition of Shimura data:

Definition 3.1. Let $G$ be a reductive $\mathbb{Q}$-algebraic group and $h: \mathbb{S} \rightarrow G_{\mathbb{R}}$ be a homomorphism. Then the pair $(G, h)$ is a Shimura datum, if:

1. The group $G^{\text {ad }}$ has no nontrivial direct compact factor over $\mathbb{Q}$.

2. The conjugation by $h(i)$ is a Cartan involution.

3. The representation ad $\circ h$ of $\mathbb{S}$ on $\operatorname{Lie}\left(G_{\mathbb{R}}\right)$ is a Hodge structure of type

$$
(1,-1),(0,0),(-1,1) \text {. }
$$

We will show that the pair $\left(\operatorname{MT}(\mathcal{X}), h_{X}\right)$ is a Shimura datum. Moreover we will determine the center of $\operatorname{Hg}(\mathcal{X})_{\mathbb{R}}$ and $\operatorname{Hg}(\mathcal{X})_{\mathbb{R}}$ in the case of a nondiscrete center. In addition we describe the monodromy in the latter case and give some examples.

Proposition 3.2. The center of $\operatorname{Hg}(\mathcal{X})(\mathbb{R})$ is given by diagonal matrices $\operatorname{diag}(\xi, \xi, \bar{\xi}, \bar{\xi})$ for $|\xi|=1$ with respect to the basis $\left\{v_{3,0}, v_{2,1}, v_{1,2}, v_{0,3}\right\}$.

Proof. Each element $Z$ in the center of $\operatorname{Hg}(\mathcal{X})(\mathbb{R})$ commutes in particular with $h_{X}\left(S^{1}\right)(\mathbb{R})$. This holds only true, if $Z$ is a diagonal matrix with respect to $\left\{v_{3,0}, v_{2,1}, v_{1,2}, v_{0,3}\right\}$ as the conjugation by elements of $h\left(S^{1}\right)(\mathbb{R})$ in Remark 1.4 shows. The subgroup of the matrices in $\operatorname{Sp}\left(H^{3}(X, \mathbb{R}), Q\right)$, which are diagonal with respect to $\left\{v_{3,0}, v_{2,1}, v_{1,2}, v_{0,3}\right\}$, is contained in $C\left(h_{W}(i)\right) \cong \mathrm{U}(2)$ and therefore compact. By Lemma 2.4, the group $\operatorname{Hg}(\mathcal{X})_{\mathbb{R}}$ cannot be compact. Thus $\mathrm{Hg}(\mathcal{X})_{\mathbb{R}}$ contains elements, which are not given by diagonal matrices with respect to $\left\{v_{3,0}, v_{2,1}, v_{1,2}, v_{0,3}\right\}$. Since $Z$ has to be real and to commute with the matrices in $\operatorname{Hg}(\mathcal{X})(\mathbb{R})$, which are not diagonal, one concludes that

$$
\begin{gathered}
Z= \pm \operatorname{diag}(\xi, 1,1, \bar{\xi}), \quad Z= \pm \operatorname{diag}(1, \xi, \bar{\xi}, 1) \\
Z=\operatorname{diag}(\xi, \xi, \bar{\xi}, \bar{\xi}) \text { or } Z=\operatorname{diag}(\xi, \bar{\xi}, \xi, \bar{\xi})
\end{gathered}
$$

with respect to the basis $\left\{v_{3,0}, v_{2,1}, v_{1,2}, v_{0,3}\right\}$. Moreover one has $|\xi|=1$, since $Z^{t} Q Z=$ $Q$. For $Z= \pm \operatorname{diag}(\xi, 1,1, \bar{\xi})$ with $\xi \neq \pm 1$ the centralizer $C(Z)$ of $Z$ in $\operatorname{Sp}\left(H^{3}(X, \mathbb{R}), Q\right)$ 
is given by the group of matrices

$$
M=\left(\begin{array}{cccc}
\zeta & 0 & 0 & 0 \\
0 & \alpha & \beta & 0 \\
0 & \bar{\beta} & \bar{\alpha} & 0 \\
0 & 0 & 0 & \bar{\zeta}
\end{array}\right) \text { with }|\zeta|=1 \text { and }\left(\begin{array}{cc}
\alpha & \beta \\
\bar{\beta} & \bar{\alpha}
\end{array}\right) \in \mathrm{SU}(1,1)
$$

as one concludes by computations using (1). Thus one concludes that $C(Z) \subset H$ from the description of $H$ in (3).

Moreover for $Z= \pm \operatorname{diag}(1, \xi, \bar{\xi}, 1)$ with $\xi \neq \pm 1$ the centralizer $C(Z)$ is given by

$$
M=\left(\begin{array}{cccc}
\alpha & 0 & 0 & \beta \\
0 & \zeta & 0 & 0 \\
0 & 0 & \bar{\zeta} & 0 \\
\bar{\beta} & 0 & 0 & \bar{\alpha}
\end{array}\right) \text { with }|\zeta|=1 \text { and }\left(\begin{array}{cc}
\alpha & \beta \\
\bar{\beta} & \bar{\alpha}
\end{array}\right) \in \mathrm{SU}(1,1)
$$

which is also a subgroup of $H$ as one concludes from analogue arguments. By Lemma 2.12 , the group $\operatorname{Hg}(\mathcal{X})_{\mathbb{R}}$ cannot be a subgroup of $H$. Since the matrices of the form

$$
\pm \operatorname{diag}(\xi, 1,1, \bar{\xi}), \quad \pm \operatorname{diag}(1, \xi, \bar{\xi}, 1) \text { with } \xi \neq \pm 1
$$

have centralizers contained in $H$, these matrices are not contained in the center of $\operatorname{Hg}(\mathcal{X})_{\mathbb{R}}$.

One can also not have that

$$
Z= \pm \operatorname{diag}(1,-1,-1,1) \in Z\left(\operatorname{Hg}(\mathcal{X})_{\mathbb{R}}\right),
$$

too, since in this case the centralizer of $Z$ in $\operatorname{Sp}\left(H^{3}(X, \mathbb{R}), Q\right)$ is $H$.

Hence one has

$$
Z=\operatorname{diag}(\xi, \xi, \bar{\xi}, \bar{\xi}) \text { or } Z=\operatorname{diag}(\xi, \bar{\xi}, \xi, \bar{\xi}) .
$$

The matrix $\operatorname{diag}(\xi, \bar{\xi}, \xi, \bar{\xi})$ commutes only with elements in $C\left(h_{X}(i)\right) \cong \mathrm{U}(2)$, if $\xi \neq$ \pm 1 . Recall that $\mathrm{U}(2)$ is compact. Moreover

$$
\operatorname{diag}(\xi, \xi, \bar{\xi}, \bar{\xi})=\operatorname{diag}(\xi, \bar{\xi}, \xi, \bar{\xi})
$$

for $\xi= \pm 1$. Again we use the fact that $\operatorname{Hg}(\mathcal{X})_{\mathbb{R}}$ cannot be compact and conclude that $Z=\operatorname{diag}(\xi, \xi, \bar{\xi}, \bar{\xi})$.

Since

$h_{X}(\xi) \in Z(\operatorname{Hg}(\mathcal{X})) \Rightarrow \operatorname{diag}(\xi, \xi, \bar{\xi}, \bar{\xi})=\operatorname{diag}\left(\xi^{3}, \xi, \bar{\xi}, \bar{\xi}^{3}\right) \Leftrightarrow \xi^{3}=\xi \Leftrightarrow \xi^{2}=1 \Leftrightarrow \xi= \pm 1$ and $h_{X}(-1)=-E_{4}$, one concludes from the previous proposition:

COROLLARY 3.3. The kernel of the representation ad $\circ h$ consists of $\{ \pm 1\}$.

Corollary 3.4. One has $\operatorname{Hg}(\mathcal{X})_{\mathbb{R}}=C\left(h_{G}(i)\right)$, if and only if $\mathrm{Hg}(\mathcal{X})_{\mathbb{R}}$ has a nondiscrete center.

Proof. Due to the fact that $C\left(h_{G}(i)\right) \cong \mathrm{U}(1,1)$ has a nondiscrete center, it is clear that $\operatorname{Hg}(\mathcal{X})_{\mathbb{R}}$ has a nondiscrete center, if $\operatorname{Hg}(\mathcal{X})_{\mathbb{R}}=C\left(h_{G}(i)\right)$. Conversely, if the center $Z\left(\operatorname{Hg}(\mathcal{X})_{\mathbb{R}}\right)$ is nondiscrete, $\operatorname{dim} Z\left(\operatorname{Hg}(\mathcal{X})_{\mathbb{R}}\right) \geq 1$. Moreover the $\mathbb{R}$-valued 
points of $Z\left(\operatorname{Hg}(\mathcal{X})_{\mathbb{R}}\right)$ are a subgroup of the group of diagonal matrices $\operatorname{diag}(\xi, \xi, \bar{\xi}, \bar{\xi})$ for $|\xi|=1$ with respect to the basis $\left\{v_{3,0}, v_{2,1}, v_{1,2}, v_{0,3}\right\}$ (see Proposition 3.2). Since the latter group is given by the onedimensional group $h_{G}\left(S^{1}\right)(\mathbb{R})$, one concludes that $Z\left(\operatorname{Hg}(\mathcal{X})_{\mathbb{R}}\right) \supseteq h_{G}\left(S^{1}\right)$. Thus $\operatorname{Hg}(\mathcal{X})_{\mathbb{R}} \subseteq C\left(h_{G}\left(S^{1}\right)\right)$. Recall that reductive groups are almost direct products of their centers and their derived subgroups (see 1.1). Moreover note that $\mathrm{Hg}(\mathcal{X})_{\mathbb{R}}$ cannot commutative. Otherwise it would be a subgroup of the compact torus

$$
C\left(h\left(S^{1}\right)\right) \cong S^{1} \times S^{1}
$$

(compare Remark 1.2), which contradicts the fact that $\mathrm{Hg}(\mathcal{X})_{\mathbb{R}}$ cannot be compact (see Lemma 2.4). Thus $\operatorname{Hg}(\mathcal{X})_{\mathbb{R}}$ has a nontrivial derived subgroup. Due to the fact that

$$
C^{\mathrm{der}}\left(h_{G}\left(S^{1}\right)\right)=C^{\mathrm{der}}\left(h_{G}(i)\right) \cong \mathrm{SU}(1,1)
$$

contains no semisimple proper subgroup and does not contain $h_{G}\left(S^{1}\right)$, one concludes $\operatorname{Hg}(\mathcal{X})_{\mathbb{R}}=C\left(h_{G}(i)\right)$

Proposition 3.5. The pair $\left(\mathrm{MT}(\mathcal{X}), h_{X}\right)$ is a Shimura datum, if $D \cong \mathbb{B}_{1}$.

Proof. By our previous results and assumptions,

$$
\operatorname{MT}^{\mathrm{ad}}(\mathcal{X})_{\mathbb{R}}=\operatorname{Hg}^{\mathrm{ad}}(\mathcal{X})_{\mathbb{R}} \cong \mathrm{PU}(1,1) .
$$

Thus $\mathrm{MT}^{\mathrm{ad}}(\mathcal{X})$ is simple and noncompact. Moreover ad $(h(i))$ yields a Cartan involution (see Lemma 1.11). Due to the fact that the conjugation by a diagonal matrix $\operatorname{diag}(a, \ldots, a)$ is the identity map, the weight homomorphism of the Hodge structure $\operatorname{ad}_{\mathrm{MT}(\mathcal{X})_{\mathbb{R}}} \circ h$ is given by $\mathbb{G}_{m, \mathbb{R}} \rightarrow\{e\}$. Thus the Hodge structure $\operatorname{ad}_{\mathrm{MT}(\mathcal{X})_{\mathbb{R}}} \circ h$ has weight zero and all characters of the representation $\operatorname{ad}_{\mathrm{MT}(\mathcal{X})_{\mathbb{R}}} \circ h$ are given by $(z / \bar{z})^{k}$ with $k \in \mathbb{Z}$. By Corollary 3.3, the kernel of ad $\left.\circ h\right|_{S^{1}}$ consists of $\{ \pm 1\}$. Since $\operatorname{dim}\left(\mathrm{MT}^{\mathrm{ad}}(\mathcal{X})_{\mathbb{R}}\right)=3$, this implies that the representation $\operatorname{ad}_{\mathrm{MT}(\mathcal{X})_{\mathbb{R}}} \circ h$ is a Hodge structure of type $(1,-1),(0,0),(-1,1)$. Thus we have a Shimura datum as claimed. $\mathrm{C}$

The variation $\mathcal{V}$ of weight 3 Hodge structures of a nonisotrivial family $\mathcal{Y} \rightarrow \mathcal{Z}$ of Calabi-Yau 3-manifolds has an underlying local system $\mathcal{V}_{\mathbb{Z}}$ corresponding to an up to conjugation unique monodromy representation

$$
\rho: \pi_{1}(\mathcal{Z}, z) \rightarrow \operatorname{GL}\left(H^{3}\left(\mathcal{Y}_{z}, \mathbb{Z}\right)\right) .
$$

Let $\mathcal{Y}_{z} \cong X$. The algebraic group $\operatorname{Mon}^{0}(\mathcal{Y})$ denotes the connected component of identity of the Zariski closure of $\rho\left(\pi_{1}(\mathcal{Z}, z)\right)$ in $\operatorname{GL}\left(H^{3}(X, \mathbb{Q})\right)$. The group $\operatorname{Mon}^{0}(\mathcal{Y})$ is a normal subgroup of $\mathrm{MT}^{\mathrm{der}}(\mathcal{Y})$, if $\mathcal{Z}$ is a connected complex algebraic manifold (see [9], Theorem 1.4). Since $\operatorname{MT}^{\mathrm{der}}(\mathcal{Y})=\operatorname{Hg}^{\text {der }}(\mathcal{Y})$ (follows from [10], Corollary 1.3.19) and $\operatorname{Sp}\left(H^{3}(X, \mathbb{Q}), Q\right)$ is simple, one concludes:

Proposition 3.6. If $\mathcal{V}_{\mathbb{Z}}$ has an infinite monodromy group, $\mathcal{Z}$ is a connected complex algebraic manifold, $\mathcal{Y}_{z} \cong X$ and

$$
\operatorname{Hg}(\mathcal{Y})=\operatorname{Sp}\left(H^{3}(X, \mathbb{Q}), Q\right),
$$

one has also

$$
\operatorname{Mon}^{0}(\mathcal{Y})=\operatorname{Sp}\left(H^{3}(X, \mathbb{Q}), Q\right) .
$$


Consider the Kuranishi family $\mathcal{X} \rightarrow B$ of $X$ and the period map

$$
p: B \rightarrow \operatorname{Grass}\left(H^{3}(X, \mathbb{C}), b_{3}(X) / 2\right)
$$

associating to each $b \in B$ the subspace

$$
F^{2}\left(H^{3}\left(\mathcal{X}_{b}, \mathbb{C}\right)\right) \subset H^{3}\left(\mathcal{X}_{b}, \mathbb{C}\right) \cong H^{3}\left(\mathcal{X}_{B}, \mathbb{C}\right) \cong H^{3}(X, \mathbb{C})
$$

as described in [14], Chapter 10. We say that $F^{2}\left(\mathcal{H}^{3}\right)_{B}$ is constant, if the period map $p: B \rightarrow \operatorname{Grass}\left(H^{3}(X, \mathbb{C}), b_{3}(X) / 2\right)$ is constant. Moreover recall that $\mathcal{Y} \rightarrow \mathcal{Z}$ is a maximal family of Calabi-Yau 3-manifolds, if $\mathcal{Z}$ can be covered by open subsets $U$ such that each $\mathcal{Y}_{U}$ is isomorphic to a Kuranishi family.

THEOREM 3.7. Assume that $\mathcal{Z}$ is a connected complex algebraic manifold and $f: \mathcal{Y} \rightarrow \mathcal{Z}$ is a maximal family of Calabi-Yau 3-manifolds with $\mathcal{Y}_{z} \cong X$ and an infinite monodromy group. Then the following statements are equivalent:

1. One has that $F^{2}\left(\mathcal{H}^{3}\right)_{B}$ is constant.

2. The monodromy representation $\rho$ of $R^{3} f_{*} \mathbb{Q}$ satisfies

$$
\rho(\gamma)\left(F^{2}\left(H^{3}(X, \mathbb{C})\right)\right)=F^{2}\left(H^{3}(X, \mathbb{C})\right) \quad\left(\forall \gamma \in \pi_{1}(\mathcal{Z}, z)\right) .
$$

3. One has

$$
\operatorname{Hg}(\mathcal{Y})_{\mathbb{R}}=C\left(h_{G}(i)\right)
$$

Proof. In [11], Section 2, we have seen that (1) implies (2).

In the case of (2) we assume that

$$
\rho(\gamma)\left(F^{2}\left(H^{3}(X, \mathbb{C})\right)\right)=F^{2}\left(H^{3}(X, \mathbb{C})\right) \text { and } \rho(\gamma)\left(H^{3}(X, \mathbb{R})\right)=H^{3}(X, \mathbb{R}) \quad\left(\forall \gamma \in \pi_{1}(\mathcal{Z}, z)\right) .
$$

Hence one has also that

$$
\rho(\gamma)\left(\overline{F^{2}\left(H^{3}(X, \mathbb{C})\right)}\right)=\overline{F^{2}\left(H^{3}(X, \mathbb{C})\right)}\left(\forall \gamma \in \pi_{1}(\mathcal{Z}, z)\right) .
$$

Thus one concludes that $h_{G}\left(S^{1}\right)$ commutes with $\operatorname{Mon}^{0}(\mathcal{Y})$. Hence $\operatorname{Mon}^{0}(\mathcal{Y})_{\mathbb{R}}$ is a semisimple group contained in the simple group $C^{\mathrm{der}}\left(h_{G}(i)\right) \cong \mathrm{SU}(1,1)$. This implies that $C^{\text {der }}\left(h_{G}(i)\right)=\operatorname{Mon}^{0}(\mathcal{Y})_{\mathbb{R}}$. Since $\operatorname{Hg}^{\text {ad }}(\mathcal{Y})=\operatorname{Hg}^{\text {ad }}(\mathcal{X})$ is simple by Theorem 2.1, we conclude

$$
C^{\operatorname{der}}\left(h_{G}(i)\right)=\operatorname{Mon}^{0}(\mathcal{Y})_{\mathbb{R}}=\operatorname{Hg}^{\operatorname{der}}(\mathcal{X})_{\mathbb{R}}
$$

from the fact that $\operatorname{Mon}^{0}(\mathcal{Y})_{\mathbb{R}}$ is a normal subgroup of $\operatorname{Hg}^{\operatorname{der}}(\mathcal{X})_{\mathbb{R}}$. Due to the fact that $h\left(S^{1}\right)$ is not contained in $C^{\mathrm{der}}\left(h_{G}(i)\right)$, the reductive group $\operatorname{Hg}(\mathcal{X})_{\mathbb{R}}$ has a nontrivial center. Thus from Corollary 3.4, we conclude (3).

Now assume that $\operatorname{Hg}(\mathcal{X})_{\mathbb{R}}=C\left(h_{G}(i)\right)$. In this case $h_{G}(i)$ commutes with the elements of $h_{b}\left(S^{1}\right)(\mathbb{R})$ for each $b \in B$. Hence $h_{G}\left(S^{1}\right)$ is contained in $C\left(h_{b}\left(S^{1}\right)\right)$. Due to the fact that $C\left(h_{b}\left(S^{1}\right)\right)$ contains only the complex structures $\pm h_{W}(i)_{b}$ and $\pm h_{G}(i)_{b}$ (see Remark 1.5), one concludes $h_{G}(i)=h_{G}(i)_{b}$ from the fact that the $V H S$ is continuous. In other terms $F^{2}\left(\mathcal{H}^{3}\right)_{B}$ is constant.

EXAMPLE 3.8. We consider an example, which ocurs in [10], 11.3.11. Let $\mathcal{E} \rightarrow$ $\mathbb{P}^{1} \backslash\{0,1, \infty\}$ denote the family of elliptic curves

$$
\mathbb{P}^{2} \supset V\left(y^{2} z-x(x-z)(x-\lambda z)\right) \rightarrow \lambda \in \mathbb{P}^{1} \backslash\{0,1, \infty\}
$$


with involution $\iota_{\mathcal{E}}$ given by $y \rightarrow-y$ over $\mathbb{P}^{1} \backslash\{0,1, \infty\}$. Moreover there is a $K 3$ surface $S$ with involution $\iota_{S}$ such that

$$
\left.\iota_{S}\right|_{H^{1,1}(S)}=\mathrm{id} \text { and }\left.\iota_{S}\right|_{H^{2,0}(S) \oplus H^{0,2}(S)}=-\mathrm{id} .
$$

By blowing up the singular sections of the family $\mathcal{E} \times S /\left\langle\left(\iota_{\mathcal{E}}, \iota_{S}\right)\right\rangle$ over $\mathbb{P}^{1} \backslash\{0,1, \infty\}$, one obtains a family $\mathcal{Y}$ of Calabi-Yau 3 -manifolds. The Hodge numbers are given by $h^{1,1}=61$ and $h^{2,1}=1$.

It is a well-known fact that the family $\mathcal{E}$ has a locally injective period map to the upper half plane. By [10], Example 1.6.9,

$$
F^{3}\left(H^{3}\left(\mathcal{Y}_{\lambda}, \mathbb{C}\right)\right)=H^{2,0}(S) \otimes H^{1,0}\left(\mathcal{E}_{\lambda}\right) \text { and } F^{2}\left(H^{3}\left(\mathcal{Y}_{\lambda}, \mathbb{C}\right)\right)=H^{2,0}(S) \otimes H^{1}\left(\mathcal{E}_{\lambda}, \mathbb{C}\right)
$$

Thus the $F^{2}$-bundle in the $V H S$ of $\mathcal{Y}$ is constant and one concludes that $\mathcal{Y}$ a maximal family from the fact that the period map associated with the $F^{3}$-bundle is locally injective. By Theorem 3.7, one concludes $\operatorname{Hg}(\mathcal{Y})_{\mathbb{R}}=C\left(h_{G}(i)\right)$.

REMARK 3.9. For the proof that $(3) \Rightarrow(1)$ in Theorem 3.7 one does not need the assumption that the base is algebraic. It is sufficient to consider the local universal deformation. Thus from [11], Section 2 one concludes that $X$ cannot occur as a fiber of a family with maximally unipotent monodromy, if $\operatorname{Hg}(\mathcal{X})_{\mathbb{R}}=C\left(h_{G}(i)\right)$.

ExAmPLE 3.10. In [11] one finds an example of a Calabi-Yau 3-manifold $X$ with Hodge numbers $h^{2,1}(X)=1$ and $h^{1,1}(X)=73$. The manifold $X$ has an automorphism $\alpha$ of degree 3 , which extends to an automorphism of $\mathcal{X}$ over $B$ and acts by a primitive cubic root of unity on $F^{2}\left(H^{3}(X, \mathbb{C})\right)$. Since $\alpha$ yields an isometry of the Hodge structure of each fiber, the generic Hodge group is contained in the centralizer $C(\alpha)$ of $\alpha$ in $\operatorname{Sp}\left(H^{3}(X, \mathbb{Q}), Q\right)$. By [11], Lemma 3.4, one has a description of $C(\alpha)_{\mathbb{R}}$ coinciding with the description of $C\left(h_{G}(i)\right)$ in Proposition 1.6. Hence $C(\alpha)_{\mathbb{R}}=C\left(h_{G}(i)\right)$. Due to the fact that $C^{\text {der }}\left(h_{G}(i)\right)$ does not contain any proper simple subgroup and $\operatorname{Hg}^{\text {der }}(\mathcal{X})_{\mathbb{R}}$ is a nontrivial simple subgroup of $C^{\operatorname{der}}\left(h_{G}(i)\right)$, one concludes $\operatorname{Hg}(\mathcal{X})_{\mathbb{R}}=C\left(h_{G}(i)\right)$.

4. The third case. Recall that $K$ denotes a maximal compact subgroup of $\operatorname{Hg}(\mathcal{X})_{\mathbb{R}}$ and that

$$
D=\operatorname{Hg}^{\operatorname{ad}}(\mathcal{X})(\mathbb{R}) / \operatorname{ad}(K(\mathbb{R}))
$$

is a Hermitian symmetric domain (see Proposition 2.7). For $D=\mathbb{B}_{1}$ we have seen that $\operatorname{Hg}(\mathcal{X})_{\mathbb{R}} \cong C\left(h_{G}(i)\right)$, if and only if $\operatorname{Hg}(\mathcal{X})$ has a nondiscrete center (see Corollary 3.4). In Section 2 we have seen that

$$
\operatorname{Hg}^{\text {ad }}(\mathcal{X})=\operatorname{Sp}^{\text {ad }}\left(H^{3}(X, \mathbb{Q}), Q\right) \text { or } \operatorname{Hg}^{\text {ad }}(\mathcal{X})_{\mathbb{R}}=\mathrm{PU}(1,1) .
$$

It remains to consider the third case that $\operatorname{Hg}(\mathcal{X})$ has a discrete center and $D \cong \mathbb{B}_{1}$. Thus assume that $\operatorname{Hg}(\mathcal{X})$ is simple and has dimension 3 . We will study $\operatorname{Hg}(\mathcal{X})_{\mathbb{R}}$ by computing its Lie algebra in this case. Let us start with the following observation:

Recall that $\operatorname{GSp}\left(H^{3}(X, \mathbb{R}), Q\right)$ is given by the matrices $M \in H^{3}(X, \mathbb{R})$ with

$$
M^{t} Q M=r Q \text { for some } r \in \mathbb{R} \text {. }
$$

Moreover recall that each representation of $\mathbb{S}$ on a real vector space $V$ is a Hodge structure by the decomposition of $V_{\mathbb{C}}$ into the eigenspaces with respect to the characters $z^{p} \bar{z}^{q}$ for $p, q \in \mathbb{Z}$ (see [4], 1.1.1). The conjugation by each diagonal matrix 
$\operatorname{diag}(a, a, a, a) \in h(\mathbb{S})(\mathbb{R})$ fixes each element of $\operatorname{GSp}\left(H^{3}(X, \mathbb{R}), Q\right)$. Thus the weight homomorphism

$$
\operatorname{ad}_{\mathrm{GSp}\left(H^{3}(X, \mathbb{R}), Q\right)} \circ h \circ w
$$

is given by $\mathbb{G}_{m, \mathbb{R}} \rightarrow\{e\}$ and the Hodge structure $\operatorname{ad}_{\mathrm{GSp}\left(H^{3}(X, \mathbb{R}), Q\right)} \circ h$ is of weight zero. Therefore the algebra $\operatorname{Lie}\left(\operatorname{GSp}\left(H^{3}(X, \mathbb{R}), Q\right)\right)_{\mathbb{C}}$ decomposes into eigenspaces with respect to the characters $(z / \bar{z})^{k}$ for $k \in \mathbb{Z}$.

4.1. Now we compute the eigenspace decomposition of $\operatorname{Lie}\left(\operatorname{Sp}\left(H^{3}(X, \mathbb{R}), Q\right)\right)$ with respect to the representation $\left(\operatorname{ad}_{\operatorname{Sp}\left(H^{3}(X, \mathbb{R}), Q\right)} \circ h_{X}\right)$ of $S^{1}$. This description is obtained from the following facts: Each of the following 3-dimensional subgroups of $\operatorname{Sp}\left(H^{3}(X, \mathbb{R}), Q\right)$ given with respect to the basis $\left\{v_{3,0}, v_{2,1}, v_{1,2}, v_{0,3}\right\}$ contains an 1dimensional subgroup on which $h\left(S^{1}\right)$ acts trivially by conjugation. Moreover the kernel of the respective restricted adjoint representation on the respective Lie algebra can be obtained from the description of the conjugation by elements of $h\left(S^{1}\right)$ in Remark 1.4. This allows us to determine the characters of the respective restricted adjoint representation, since we have only characters of the type $(z / \bar{z})^{k}$ for $k \in \mathbb{Z}$ as we have seen above. Since

$$
10=\operatorname{dim} \operatorname{Sp}\left(H^{3}(X, \mathbb{R}), Q\right),
$$

one checks easily that one can find a basis of eigenvectors by the computations below:

- The centralizer $C\left(h\left(S^{1}\right)\right)$ is a twodimensional torus (see Remark 1.5), which yields a corresponding twodimensional eigenspace with character 1.

- The group $C^{\operatorname{der}}\left(h_{W}(i)\right)$ is given by the matrices

$$
M=\left(\begin{array}{cccc}
\alpha & 0 & \beta & 0 \\
0 & \bar{\alpha} & 0 & -\beta \\
-\bar{\beta} & 0 & \bar{\alpha} & 0 \\
0 & \bar{\beta} & 0 & \alpha
\end{array}\right) \text { with }|\alpha|^{2}-|\beta|^{2}=1
$$

(this follows from Proposition 1.7 and Remark 1.8). The complexified Lie algebra of $C^{\operatorname{der}}\left(h_{W}(i)\right)$ has an eigenspace with character $(\bar{z} / z)^{2}$ and an eigenspace with character $(z / \bar{z})^{2}$.

- The group $C^{\operatorname{der}}\left(h_{G}(i)\right)$ is given by the matrices

$$
M=\left(\begin{array}{cccc}
\alpha & \beta & 0 & 0 \\
\bar{\beta} & \bar{\alpha} & 0 & 0 \\
0 & 0 & \bar{\alpha} & \bar{\beta} \\
0 & 0 & \beta & \alpha
\end{array}\right) \text { with }|\alpha|^{2}-|\beta|^{2}=1
$$

(this follows from Proposition 1.6 and Remark 1.8). The complexified Lie algebra of $C^{\operatorname{der}}\left(h_{G}(i)\right)$ has an eigenspace with character $\bar{z} / z$ and an eigenspace with character $z / \bar{z}$.

- By explicit computations using the definition of $Q$ (see (1)), one can easily check that the group $C G$ given by the matrices

$$
M=\left(\begin{array}{cccc}
1 & 0 & 0 & 0 \\
0 & \alpha & \beta & 0 \\
0 & \bar{\beta} & \bar{\alpha} & 0 \\
0 & 0 & 0 & 1
\end{array}\right) \text { with } \operatorname{det}(M)=1
$$


is a subgroup of $\operatorname{Sp}\left(H^{3}(X, \mathbb{R}), Q\right)$. The complexified Lie algebra of the group $C G$ has an eigenspace with character $\bar{z} / z$ and an eigenspace with character $z / \bar{z}$.

- By explicit computations using the definition of $Q$ (see (1)), one can easily check that the group given by the matrices

$$
M=\left(\begin{array}{cccc}
\alpha & 0 & 0 & \beta \\
0 & 1 & 0 & 0 \\
0 & 0 & 1 & 0 \\
\bar{\beta} & 0 & 0 & \bar{\alpha}
\end{array}\right) \text { with } \operatorname{det}(M)=1
$$

is a subgroup of $\operatorname{Sp}\left(H^{3}(X, \mathbb{R}), Q\right)$. The complexified Lie algebra of this group has an eigenspace with character $(\bar{z} / z)^{3}$ and an eigenspace with character $(z / \bar{z})^{3}$.

From now on we make computations with respect to the basis $\left\{v_{3,0}, v_{2,1}, v_{1,2}, v_{0,3}\right\}$. The Lie algebra of $\operatorname{Hg}(\mathcal{X})_{\mathbb{R}}$ contains clearly the vector space

$$
\operatorname{Lie}\left(h_{X}\left(S^{1}\right)\right)=\operatorname{Span}_{\mathbb{R}}(\operatorname{diag}(3 i, i,-i,-3 i)) .
$$

Recall that the representation ad $\circ h_{X}$ of $S^{1}$ on $\operatorname{Lie}(\operatorname{Hg}(\mathcal{X}))$ is a weight zero Hodge structure of type $(1,-1),(0,0),(-1,1)$ (follows from Proposition 3.5) and the maximal torus of the 3 -dimensional simple group $\operatorname{Hg}(\mathcal{X})_{\mathbb{R}}$ has dimension 1 . The direct sum of the eigenspaces with the characters $1, z / \bar{z}$ and $\bar{z} / z$ coincides with

$$
\operatorname{Lie}\left(C^{\operatorname{der}}\left(h_{G}(i)\right)\right)_{\mathbb{C}} \oplus \operatorname{Lie}(C G)_{\mathbb{C}}
$$

as one concludes from 4.1. Hence

$$
\operatorname{Lie}(\operatorname{Hg}(\mathcal{X})) \subset \operatorname{Lie}\left(C^{\text {der }}\left(h_{G}(i)\right)\right) \oplus \operatorname{Lie}(C G) .
$$

Moreover recall that $\operatorname{Lie}\left(\operatorname{Hg}(\mathcal{X})_{\mathbb{R}}\right) \cong \mathfrak{s u}(1,1)$, where

$\mathfrak{s u}(1,1)=\operatorname{Span}_{\mathbb{R}}(H, X, Y)$ for $H=\left(\begin{array}{cc}i & 0 \\ 0 & -i\end{array}\right), X=\left(\begin{array}{cc}0 & 1 \\ 1 & 0\end{array}\right), Y=\left(\begin{array}{cc}0 & i \\ -i & 0\end{array}\right)$

(compare Remark 1.9). One computes easily that

$$
[H, X]=2 Y, \quad[Y, H]=2 X, \quad[Y, X]=2 H .
$$

Moreover $H$ generates the Lie subalgebra of a maximal torus of $\operatorname{Hg}(\mathcal{X})_{\mathbb{R}}$ with respect to the identification above. Thus $\operatorname{Span}(H)=\operatorname{Lie}\left(h_{X}\left(S^{1}\right)\right)$. Since

$[H, X-i Y]=2 Y+2 i X=2 i(X-i Y)$ and $[H, X+i Y]=2 Y-2 i X=-2 i(X-i Y)$,

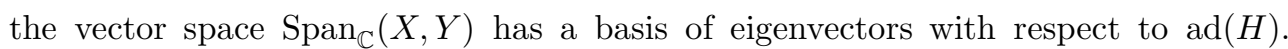
Therefore each $M \in \operatorname{Span}_{\mathbb{R}}(X, Y) \subset \operatorname{Lie}(\operatorname{Hg}(\mathcal{X}))$ has the form

$$
M=\left(\begin{array}{cccc}
0 & * & 0 & 0 \\
* & 0 & * & 0 \\
0 & * & 0 & * \\
0 & 0 & * & 0
\end{array}\right) \in \operatorname{Lie}\left(C^{\operatorname{der}}\left(h_{G}(i)\right)\right)+\operatorname{Lie}(C G)
$$


where $C G$ was introduced in 4.1. The explicit descriptions of $C^{\mathrm{der}}\left(h_{G}(i)\right)$ and $C G$ in 4.1 and the explicit description of $\mathrm{SU}(1,1)$ in Remark 1.8, yield natural isomorphisms

$$
C^{\mathrm{der}}\left(h_{G}(i)\right) \cong C G \cong \mathrm{SU}(1,1) .
$$

Thus from the explicit description of $\mathfrak{s u}(1,1)$ in Remark 1.9, we conclude

$$
M=\left(\begin{array}{cccc}
0 & x & 0 & 0 \\
\bar{x} & 0 & y & 0 \\
0 & \bar{y} & 0 & x \\
0 & 0 & \bar{x} & 0
\end{array}\right)
$$

for some $x, y \in \mathbb{C}$. One has an $M \in \operatorname{Lie}(\operatorname{Hg}(\mathcal{X}))$ with $x \neq 0$. Otherwise one would have

$$
N_{1}=\left(\begin{array}{cccc}
0 & 0 & 0 & 0 \\
0 & 0 & 1 & 0 \\
0 & 1 & 0 & 0 \\
0 & 0 & 0 & 0
\end{array}\right), \quad N_{2}=\left(\begin{array}{cccc}
0 & 0 & 0 & 0 \\
0 & 0 & i & 0 \\
0 & -i & 0 & 0 \\
0 & 0 & 0 & 0
\end{array}\right) \in \operatorname{Lie}(\operatorname{Hg}(\mathcal{X}))
$$

since $\operatorname{dim} \operatorname{Span}_{\mathbb{R}}(X, Y)=2$. This implies

$$
\left[N_{1}, N_{2}\right]=\operatorname{diag}(0,-2 i, 2 i, 0) \neq 0 .
$$

But this cannot hold true, since $\operatorname{Span}_{\mathbb{R}}(\operatorname{diag}(3 i, i,-i,-3 i))$ is the subvector space of diagonal matrices in $\operatorname{Lie}\left(\operatorname{Hg}(\mathcal{X})_{\mathbb{R}}\right)$. Moreover one has

$$
\left[\left(\begin{array}{cccc}
0 & x & 0 & 0 \\
\bar{x} & 0 & y & 0 \\
0 & \bar{y} & 0 & x \\
0 & 0 & \bar{x} & 0
\end{array}\right),\left(\begin{array}{cccc}
0 & 0 & 0 & 0 \\
0 & 0 & z & 0 \\
0 & \bar{z} & 0 & 0 \\
0 & 0 & 0 & 0
\end{array}\right)\right]=\left(\begin{array}{cccc}
0 & 0 & x z & 0 \\
0 & y \bar{z}-z \bar{y} & 0 & -z x \\
-\bar{x} \bar{z} & 0 & \bar{y} z-\bar{z} y & 0 \\
0 & \bar{x} \bar{z} & 0 & 0
\end{array}\right) \notin \operatorname{Lie}(\operatorname{Hg}(\mathcal{X}))
$$

for $x, z \neq 0$. Hence we conclude:

Proposition 4.2. Assume that $\mathrm{Hg}^{\text {ad }}(\mathcal{X})_{\mathbb{R}} \cong \mathrm{PU}(1,1)$ and $\mathrm{Hg}(\mathcal{X})$ has a discrete center. Then for some $x, y \in \mathbb{C}$ we have

$$
\operatorname{Lie}(\operatorname{Hg}(\mathcal{X}))=\operatorname{Span}_{\mathbb{R}}\left(\left(\begin{array}{cccc}
3 i & 0 & 0 & 0 \\
0 & i & 0 & 0 \\
0 & 0 & -i & 0 \\
0 & 0 & 0 & -3 i
\end{array}\right),\left(\begin{array}{cccc}
0 & 1 & 0 & 0 \\
1 & 0 & x & 0 \\
0 & \bar{x} & 0 & 1 \\
0 & 0 & 1 & 0
\end{array}\right),\left(\begin{array}{cccc}
0 & i & 0 & 0 \\
-i & 0 & y & 0 \\
0 & \bar{y} & 0 & i \\
0 & 0 & -i & 0
\end{array}\right)\right) .
$$

Now we determine the possible choices of $x, y \in \mathbb{C}$ :

(6)

$$
\begin{aligned}
& {\left[\left(\begin{array}{cccc}
0 & i & 0 & 0 \\
-i & 0 & y & 0 \\
0 & \bar{y} & 0 & i \\
0 & 0 & -i & 0
\end{array}\right),\left(\begin{array}{cccc}
0 & 1 & 0 & 0 \\
1 & 0 & x & 0 \\
0 & \bar{x} & 0 & 1 \\
0 & 0 & 1 & 0
\end{array}\right)\right]} \\
& =\left(\begin{array}{cccc}
2 i & 0 & i x-y & 0 \\
0 & -2 i+\bar{x} y-x \bar{y} & 0 & y-i x \\
\bar{y}+i \bar{x} & 0 & 2 i+x \bar{y}-\bar{x} y & 0 \\
0 & -i \bar{x}-\bar{y} & 0 & -2 i
\end{array}\right)
\end{aligned}
$$

Hence one obtains

$$
i x-y=0 \Leftrightarrow i x=y \Leftrightarrow \Im(y)=\Re(x), \quad \Re(y)=-\Im(x) .
$$


Thus the matrix on the right hand side of (6) is contained in $\operatorname{Span}(\operatorname{diag}(3 i, i,-i,-3 i))$ and for the second entry in the second column we obtain

$$
-2 i+\bar{x} y-x \bar{y}=\frac{2}{3} i \Rightarrow \bar{x} y-x \bar{y}=\frac{8}{3} i
$$

We have independent of the choice of $x$ and $y$ that

$$
\Re(\bar{x} y-x \bar{y})=\Re(\bar{x} y-\overline{\bar{x} y})=0
$$

The previous equations imply:

$$
\begin{aligned}
\frac{8}{3}=\Im(\bar{x} y-x \bar{y}) & =-\Im(x) \Re(y)+\Re(x) \Im(y)+\Re(x) \Im(y)-\Im(x) \Re(y) \\
& =2 \Re(x)^{2}+2 \Im(x)^{2}=2|x|^{2} .
\end{aligned}
$$

By using $i x=y$, we compute

$$
\begin{aligned}
& {\left[\left(\begin{array}{cccc}
0 & i & 0 & 0 \\
-i & 0 & y & 0 \\
0 & \bar{y} & 0 & i \\
0 & 0 & -i & 0
\end{array}\right),\left(\begin{array}{cccc}
3 i & 0 & 0 & 0 \\
0 & i & 0 & 0 \\
0 & 0 & -1 & 0 \\
0 & 0 & 0 & -3 i
\end{array}\right)\right]=\left(\begin{array}{cccc}
0 & 2 & 0 & 0 \\
2 & 0 & 2 x & 0 \\
0 & 2 \bar{x} & 0 & 2 \\
0 & 0 & 2 & 0
\end{array}\right)} \\
& \text { and }\left[\left(\begin{array}{cccc}
3 i & 0 & 0 & 0 \\
0 & i & 0 & 0 \\
0 & 0 & -1 & 0 \\
0 & 0 & 0 & -3 i
\end{array}\right),\left(\begin{array}{cccc}
0 & 1 & 0 & 0 \\
1 & 0 & x & 0 \\
0 & \bar{x} & 0 & 1 \\
0 & 0 & 1 & 0
\end{array}\right)\right]=\left(\begin{array}{cccc}
0 & 2 i & 0 & 0 \\
-2 i & 0 & 2 y & 0 \\
0 & 2 \bar{y} & 0 & 2 i \\
0 & 0 & -2 i & 0
\end{array}\right)
\end{aligned}
$$

By the same arguments as in the proof of Lemma 1.2, we conclude that a Lie subgroup of $\mathrm{GL}_{n}(\mathbb{R})$ isomorphic to $\mathrm{SU}(1,1)(\mathbb{R})$ is the group of $\mathbb{R}$-valued points of a group isomorphic to $\mathrm{SU}(1,1)$. Note that that the center of $\mathrm{SU}(1,1)$ consists of $\{ \pm 1\}$. Since for each $x \in \mathbb{C}$ with $|x|=\frac{2}{\sqrt{3}}$ there is a Lie algebra isomorphic to $\mathfrak{s u}(1,1)$, which has an associated subgroup of $\operatorname{GL}\left(H^{3}(X, \mathbb{R})\right)$ with center consisting of $\{ \pm 1\}$, we conclude:

Proposition 4.3. For each $x \in \mathbb{C}$ with $|x|=\frac{2}{\sqrt{3}}$ there is a simple $\mathbb{R}$-algebraic subgroup

$$
G_{x} \subset \operatorname{Sp}\left(H^{3}(X, \mathbb{R}), Q\right)
$$

isomorphic to $\mathrm{SL}_{\mathbb{R}}(2)$ such that $h\left(S^{1}\right) \subset G_{x}$.

COROLlary 4.4. In the third case we have

$$
\operatorname{Hg}(\mathcal{X})_{\mathbb{R}} \cong \mathrm{SL}_{\mathbb{R}}(2)
$$

and all representations of generic Hodge groups of third type on $H^{3}(X, \mathbb{R})$ are isomorphic.

We will see that in the third case the representation of $\operatorname{Hg}(\mathcal{X})_{\mathbb{R}}$ on $H^{3}(X, \mathbb{R})$ is isomorphic to the natural representation of $\mathrm{SL}_{\mathbb{R}}(2)$ on $\operatorname{Sym}^{3}\left(\mathbb{R}^{2}\right)$ later.

LEMMA 4.5. Each unipotent matrix in $G_{x}$ has a Jordan block of length $\geq 3$. 
Proof. A unipotent matrix in $G_{x}$, whose Jordan blocks have the maximal length 2 , would correspond to a matrix $M \in \operatorname{Lie}\left(G_{x}\right)$, whose square is zero. One has that

$$
\left(m_{i, j}\right)=M^{2}=\left(\begin{array}{cccc}
a 3 i & c+b i & 0 & 0 \\
c-b i & a i & c x+b y & 0 \\
0 & c \bar{x}+b \bar{y} & -a i & c+b i \\
0 & 0 & c-b i & -3 a i
\end{array}\right)^{2}=0
$$

with $a, b, c \in \mathbb{R}$ is satisfied, only if

$$
m_{1,2}=4 a i(c+b i)=0 .
$$

Hence $a=0$ or $c+b i=0$. The reader checks easily that $M^{2}$ cannot be zero in either case with the exception given by $M=0$.

EXAMPLE 4.6. In [5] there is a list of explicitly computed examples of variations of Hodge structures of families $\mathcal{Y} \rightarrow \mathbb{P}^{1} \backslash\{0,1, \infty\}$ of Calabi-Yau 3-manifolds with 1-dimensional complex moduli. Note that each of these variations has a monodromy group containing a unipotent matrix, which has only Jordan blocks of length $\leq 2$. Due to the fact that $\operatorname{Mon}^{0}(\mathcal{Y}) \subseteq \operatorname{Hg}(\mathcal{Y})$, we conclude from Lemma 4.5 that there is no $x$ with $|x|=\frac{2}{\sqrt{3}}$ such that $\operatorname{Hg}(\mathcal{Y})_{\mathbb{R}} \cong G_{x}$. Moreover each example in [5] has maximally unipotent monodromy. Thus we are not in the case $\operatorname{Hg}(\mathcal{Y})_{\mathbb{R}}=C\left(h_{G}(i)\right)$ for these examples. Therefore the examples of [5] have a generic Hodge group given by $\operatorname{Sp}\left(H^{3}(Y, \mathbb{Q}), Q\right)$, where $Y$ denotes an arbitrary fiber of the respective family $\mathcal{Y}$.

It would be very nice to find an example for the third case $\operatorname{Hg}(\mathcal{X})_{\mathbb{R}}=G_{x}$. At present there is no example of a family of Calabi-Yau manifolds with 1-dimensional complex moduli known to the author, which satisfies the third case. Nevertheless one finds a Calabi-Yau like variation of Hodge structures of third case, which arises in a natural way over a curve as we will see now(for the definition see 4.10. For this example one uses the construction of C. Borcea [1]:

Construction 4.7. Let $E_{1}, E_{2}, E_{3}$ be elliptic curves with involutions $\iota_{1}, \iota_{2}, \iota_{3}$ such that $E_{j} / \iota_{j} \cong \mathbb{P}^{1}$. The singular variety

$$
E_{1} \times E_{2} \times E_{3} /\left\langle\left(\iota_{1}, \iota_{2}\right),\left(\iota_{2}, \iota_{3}\right)\right\rangle
$$

yields a Calabi-Yau 3-manifold $C$ by blowing up the singularities. The isomorphism class of $C$ depends on the choice of the sequence of blowing ups. Nevertheless the Hodge structure on $H^{3}(C, \mathbb{Z})$ does not depend on the choice of this sequence and is given by the tensor product

$$
H^{3}(C, \mathbb{C})=H^{1}\left(E_{1}, \mathbb{C}\right) \otimes H^{1}\left(E_{2}, \mathbb{C}\right) \otimes H^{1}\left(E_{3}, \mathbb{C}\right)
$$

of the respective Hodge structures.

Let $f_{1}: \mathcal{E} \rightarrow \mathbb{A}^{1} \backslash\{0,1\}$ denote the family of elliptic curves given by

$$
\mathbb{P}^{2} \supset V\left(y^{2} z=x(x-z)(x-\lambda z)\right) \rightarrow \lambda \in \mathbb{A}^{1} \backslash\{0,1\} .
$$

By using the involution of $\mathcal{E}$ over $\mathbb{A}^{1} \backslash\{0,1\}$ and three copies of $\mathcal{E} \rightarrow \mathbb{A}^{1} \backslash\{0,1\}$, one can give a relative version of the previous construction. Let $f_{3}: \mathcal{C} \rightarrow\left(\mathbb{A}^{1} \backslash\{0,1\}\right)^{3}$ denote a family obtained by this relative version of $C$. Borcea's construction. 
Recall that a Calabi-Yau 3-manifold $X$ has complex multiplication $(C M)$, if the Hodge group $\operatorname{Hg}\left(H^{3}(X, Q), h\right)$ is a torus. For $\operatorname{Hg}(\mathcal{X})_{\mathbb{R}}=C\left(h_{G}(i)\right)$ the pair is a Shimura datum (see Proposition 3.5). Thus we have a dense set of $C M$ fibers. ${ }^{4}$ But in this case one cannot have maximally unipotent monodromy (see Remark 3.9). Moreover for $\operatorname{Hg}(\mathcal{X})=\operatorname{Sp}\left(H^{3}(X, \mathbb{Q}), Q\right)$ the associated Hermitian symmetric domain has a dimension larger than the dimension of the basis. For this case one conjectures that only finitely many $C M$ fibers occur. Hence for families of Calabi-Yau 3-manifolds with onedimensional complex moduli it is feasible to concjecture that the existence of infinitely many nonisomorphic $C M$ fibers and maximally unipotent monodromy exclude each other. This does not hold true for Calabi-Yau 3-manifolds with higher dimensional complex moduli, since the family $f_{3}: \mathcal{C} \rightarrow\left(\mathbb{A}^{1} \backslash\{0,1\}\right)^{3}$ has maximally unipotent monodromy and a dense set of $C M$ fibers:

REMARK 4.8. Let $\Delta^{*}$ denote the punctured disc. One finds a neighbourhood $U$ of the point $(0,0,0) \in \mathbb{A}^{3}$ such that $\mathcal{C}$ is locally defined over $\left(\Delta^{*}\right)^{3} \subset U$. Let $D_{1}, D_{2}, D_{3}$ denote the irreducible components of the complement of $\left(\Delta^{*}\right)^{3} \subset U$ and $\gamma_{i}$ denote a closed path given by a loop around $D_{i}$. The family $f_{1}: \mathcal{E} \rightarrow \mathbb{A}^{1} \backslash\{0,1\}$ of elliptic curves has unipotent monodromy around 0 with

$$
\rho(\gamma)=\left(\begin{array}{ll}
1 & 2 \\
0 & 1
\end{array}\right)
$$

with respect to a basis $\{a, b\}$ (follows from the computations in [10], Section 3.3). Thus one computes easily that

$N_{r, s, t}=r \log \rho\left(\gamma_{1}\right)+s \log \rho\left(\gamma_{2}\right)+t \log \rho\left(\gamma_{3}\right)=\left(\begin{array}{cccccccc}0 & 2 t & 2 s & 0 & 2 r & 0 & 0 & 0 \\ 0 & 0 & 0 & 2 s & 0 & 2 r & 0 & 0 \\ 0 & 0 & 0 & 2 t & 0 & 0 & 2 r & 0 \\ 0 & 0 & 0 & 0 & 0 & 0 & 0 & 2 r \\ 0 & 0 & 0 & 0 & 0 & 2 t & 2 s & 0 \\ 0 & 0 & 0 & 0 & 0 & 0 & 0 & 2 s \\ 0 & 0 & 0 & 0 & 0 & 0 & 0 & 2 t \\ 0 & 0 & 0 & 0 & 0 & 0 & 0 & 0\end{array}\right)$

with respect to the basis

$$
\begin{aligned}
& \mathcal{B}=\left\{a_{1} \otimes a_{2} \otimes a_{3}, \quad a_{1} \otimes a_{2} \otimes b_{3}, \quad a_{1} \otimes b_{2} \otimes a_{3}, \quad a_{1} \otimes b_{2} \otimes b_{3},\right. \\
& \left.b_{1} \otimes a_{2} \otimes a_{3}, \quad b_{1} \otimes a_{2} \otimes b_{3}, \quad b_{1} \otimes b_{2} \otimes a_{3}, \quad b_{1} \otimes b_{2} \otimes b_{3}\right\} .
\end{aligned}
$$

By analogue computations, one gets the same result for all maximal-depth normal crossing points of $\left(\mathbb{A}^{1} \backslash\{0,1\}\right)^{3}$. Thus the family $\mathcal{C} \rightarrow\left(\mathbb{A}^{1} \backslash\{0,1\}\right)^{3}$ has maximally unipotent monodromy around each maximal-depth normal crossing point (for the definition of maximally unipotent monodromy see [8]). Moreover $C$ has $C M$, if and only if $E_{1}, E_{2}, E_{3}$ have $C M$ as complex tori (see [1], Proposition 3.1). Since it is a well-known fact that $\mathcal{E}$ has a dense set of fibers $\mathcal{E}_{\lambda}$ such that $\mathcal{E}_{\lambda}$ has $C M$, one concludes that $\mathcal{C}$ has a dense set of $C M$ fibers.

Now we come to the Calabi-Yau like $V H S$ of third type. Let $\Delta \subset\left(\mathbb{A}^{1} \backslash\{0,1\}\right)^{3}$ be the diagonal obtained from the closed embedding

$$
\mathbb{A}^{1} \backslash\{0,1\} \hookrightarrow\left(\mathbb{A}^{1} \backslash\{0,1\}\right)^{3} \text { via } x \rightarrow(x, x, x)
$$

\footnotetext{
${ }^{4}$ The proof uses arguments, which occur already in [11], Section 4 . One has only to replace $C(\alpha)$ by $\operatorname{Hg}(\mathcal{X})$ and use the same arguments, which occur after the proof of [11], Lemma 4.
} 
As we will see the rational $V H S$ of the restricted family $\mathcal{C}_{\Delta} \rightarrow \Delta$ contains a sub- $V H S$ of third type. Let

$$
\mathcal{H}^{1}=R^{1}\left(f_{1}\right)_{*} \mathbb{Q} \otimes \mathcal{O}_{\Delta} \text { and } \mathcal{H}^{3}=R^{3}\left(\left.f_{3}\right|_{\mathcal{C}_{\Delta}}\right)_{*} \mathbb{Q} \otimes \mathcal{O}_{\Delta} .
$$

4.9. One has that $\mathcal{H}^{3}=\left(\mathcal{H}^{1}\right)^{\otimes 3}$ (see also [13], Remark 7.4) and $F^{3}\left(\mathcal{H}^{3}\right.$ ) is contained in the symmetric product $\operatorname{Sym}^{3}\left(\mathcal{H}^{1}\right)$. Hence

$$
H^{3,0}\left(\mathcal{C}_{(\lambda, \lambda, \lambda)}\right), H^{0,3}\left(\mathcal{C}_{(\lambda, \lambda, \lambda)}\right) \subset \operatorname{Sym}^{3}\left(H^{1}\left(\mathcal{E}_{\lambda}, \mathbb{C}\right)\right)
$$

for each $(\lambda, \lambda, \lambda) \in \Delta$. Since $F^{3}\left(\mathcal{H}^{3}\right) \subset \operatorname{Sym}^{3}\left(\mathcal{H}^{1}\right)$, one obtains $\nabla_{t} \omega(b) \in$ $\operatorname{Sym}\left(H^{1}\left(\mathcal{E}_{\lambda}, \mathbb{Q}\right)\right)$ for each section $\omega \in F^{3}\left(\mathcal{H}_{\Delta}^{3}\right)(U)$ and $t \in T_{b} \Delta$. By Bryant-Griffiths [2], one has that $F^{2}\left(\mathcal{H}^{3}\right)$ is generated by the sections of $F^{3}\left(\mathcal{H}^{3}\right)$ and their differentials. Therefore one concludes that $F^{2}\left(\mathcal{H}^{3}\right) \cap \operatorname{Sym}^{3}\left(\mathcal{H}^{1}\right)$ is of rank 2 and we have a polarized rational variation $\mathcal{V}$ of Hodge structures of type

$$
(3,0), \quad(2,1), \quad(1,2), \quad(0,3)
$$

with the underlying local system $\operatorname{Sym}^{3}\left(R^{1}\left(f_{1}\right)_{*} \mathbb{Q}\right)$ of rank 4. This $V H S$ satisfies that $F^{2}(\mathcal{V})$ is generated by the sections of $F^{3}(\mathcal{V})$ and their differentials along $\Delta$, and that $F^{1}(\mathcal{V})=F^{3}(\mathcal{V})^{\perp}$ with respect to the polarization. By [2], these two properties characterize the $V H S$ of a family of Calabi-Yau 3-manifolds. In this sense $\mathcal{V}$ is a Calabi-Yau like sub- $V H S$ of the rational $V H S$ of $\mathcal{C}_{\Delta}$.

4.10. Let $M$ be connected complex manifold and $\mathcal{W} \rightarrow M$ be a Calabi-Yau like VHS with

$$
h^{3,0}\left(\mathcal{W}_{m}\right)=h^{2,1}\left(\mathcal{W}_{m}\right)=h^{1,2}\left(\mathcal{W}_{m}\right)=h^{0,3}\left(\mathcal{W}_{m}\right)=1
$$

for each $m \in M$. We say that $\mathcal{W}$ is of third type, if the center of its generic Hodge group is discrete and the associated Hermitian symmetric domain is $\mathbb{B}_{1}$. Note that all previous arguments are also valid for a Calabi-Yau like VHS in the sense of [2], which is not necessarily the $V H S$ of a family of Calabi-Yau 3-manifolds. Thus there is an $x \in \mathbb{C}$ with $|x|=\frac{2}{\sqrt{3}}$ such that $\operatorname{Hg}(\mathcal{W})_{\mathbb{R}}=G_{x}$ for a Calabi-Yau like $V H S$ of third type.

Let $E$ be an elliptic curve and $M \in \mathrm{GL}\left(H^{1}(E, \mathbb{Q})\right)$ be given by

$$
M=\left(\begin{array}{ll}
a & b \\
c & d
\end{array}\right) \in \mathrm{GL}\left(H^{1}(E, \mathbb{Q})\right)
$$

with respect to a basis $\left\{e_{1}, e_{2}\right\}$ of $H^{1}(E, \mathbb{Q})$. Moreover let

$$
K r^{3}(M)=M \otimes M \otimes M
$$

denote the third Kronecker power of $M$. One can easily check that

$$
K r^{3}(M)\left(\operatorname{Sym}^{3}\left(H^{1}(E, \mathbb{Q})\right)\right)=\operatorname{Sym}^{3}\left(H^{1}(E, \mathbb{Q})\right)
$$

for each $M \in \mathrm{GL}\left(H^{1}(E, \mathbb{Q})\right)$. Moreover one can easily compute that $K r(M)$ acts on $\operatorname{Sym}^{3}\left(H^{1}(E, \mathbb{Q})\right)$ by the matrix

$$
r(M)=\left(\begin{array}{cccc}
a^{3} & 3 a^{2} b & 3 a b^{2} & b^{3} \\
a^{2} c & a^{2} d+2 a b c & 2 a b d+b^{2} c & b^{2} d \\
a c^{2} & a c d+b c^{2} & a d^{2}+2 b c d & b d^{2} \\
c^{3} & 3 c^{2} d & 3 c d^{2} & d^{3}
\end{array}\right)
$$


with respect to the basis

$$
\begin{aligned}
& \left\{e_{1} \otimes e_{1} \otimes e_{1}, \quad e_{1} \otimes e_{1} \otimes e_{2}+e_{1} \otimes e_{2} \otimes e_{1}+e_{2} \otimes e_{1} \otimes e_{1},\right. \\
& \left.e_{1} \otimes e_{2} \otimes e_{2}+e_{2} \otimes e_{1} \otimes e_{2}+e_{1} \otimes e_{2} \otimes e_{2}, \quad e_{2} \otimes e_{2} \otimes e_{2}\right\} .
\end{aligned}
$$

LEMMA 4.11. One has the homomorphisms

$$
r: \mathrm{GL}\left(H^{1}(E, \mathbb{Q})\right) \rightarrow \mathrm{GL}\left(\operatorname{Sym}^{3}\left(H^{1}(E, \mathbb{Q})\right)\right)
$$

and

$$
\left.r\right|_{\mathrm{SL}\left(H^{1}(E, \mathbb{Q})\right)}: \mathrm{SL}\left(H^{1}(E, \mathbb{Q})\right) \rightarrow \mathrm{SL}\left(\operatorname{Sym}^{3}\left(H^{1}(E, \mathbb{Q})\right)\right)
$$

of $\mathbb{Q}$-algebraic groups.

Proof. From (7) one concludes that $r$ is an regular map. Note that the determinant of $r(M)$ is given by $\operatorname{det}^{6}(M)$ for each $M \in \mathrm{GL}\left(H^{1}(E, \mathbb{Q})\right)$. This follows by computing $\operatorname{det}\left(r\left(J_{M}\right)\right)$, where $J_{M}$ denotes the associated Jordan form of $M$. Since one can easily check that $K r^{3}$ respects the matrix multiplication, one concludes that the same holds true for $r$. Thus we obtain the homomorphisms of $\mathbb{Q}$-algebraic groups as claimed.

Let $G$ denote the Zariski closure of $r\left(\operatorname{SL}\left(H^{1}(E, \mathbb{Q})\right)\right)$ in $\operatorname{GL}\left(\operatorname{Sym}^{3}\left(H^{1}(E, \mathbb{Q})\right)\right)$. It is a well-known fact that $G$ is an algebraic group.

Lemma 4.12. The group $G$ has at most dimension 3.

Proof. Let

$$
M=\left(\begin{array}{ll}
a & b \\
c & d
\end{array}\right) \in \mathrm{GL}\left(H^{1}(E, \mathbb{Q})\right) .
$$

For $\left(m_{i, j}\right)=r(M)$ one has that

$$
\begin{aligned}
m_{2,2}^{3} & =(a(a d+2 b c))^{3} \\
& =a^{3}\left(a^{3} d^{3}+6 a^{2} b c d^{2}+12 a b^{2} c^{2} d+8 b^{3} c^{3}\right) \\
& =m_{1,1}\left(m_{1,1} m_{4,4}+\frac{2}{3} m_{1,2} m_{4,3}+\frac{4}{3} m_{1,3} m_{4,2}+8 m_{1,4} m_{4,1}\right)
\end{aligned}
$$

(follows from (7)). In an analogue way one can express $m_{2,3}^{3}, m_{3,2}^{3}, m_{3,3}^{3}$ by equations with entries $m_{i, j}$ such that $\{i, j\} \cap\{1,4\} \neq \emptyset$. Note that for all other entries $m_{i, j}$ of $r(M)$ such that $\{i, j\} \cap\{1,4\} \neq \emptyset$ the power $m_{i, j}^{3}$ satisfies some equation in terms of

$$
m_{1,1}=a^{3}, m_{1,8}=b^{3}, m_{8,1}=c^{3}, m_{8,8}=d^{3}
$$

(compare (7)). Due to these facts, one finds enough equations such that the Zariski closure $\overline{r\left(\mathrm{GL}\left(H^{1}(E, \mathbb{Q})\right)\right.}$ of the group $r\left(\mathrm{GL}\left(H^{1}(E, \mathbb{Q})\right)(\mathbb{Q})\right.$ has at most dimension 4 . Since $\operatorname{det}(r(M))=\operatorname{det}^{6}(M)$, the set on the right hand site of the inequality

$$
\left.G^{0} \subseteq \overline{\left(r \left(\mathrm{GL}\left(H^{1}(E, \mathbb{Q})\right)\right.\right.} \cap \mathrm{SL}\left(\operatorname{Sym}^{3} H^{1}(E, \mathbb{Q})\right)\right)^{0}
$$

is a proper Zariski closed subset of $\overline{r\left(\mathrm{GL}\left(H^{1}(E, \mathbb{Q})\right)\right.}$. Thus one concludes that

$$
\operatorname{dim} G \leq 3
$$


Note that the Hodge structure of $\mathcal{C}_{(\lambda, \lambda, \lambda)}$ is given by the tensor product $H^{1}\left(\mathcal{E}_{\lambda}, \mathbb{Q}\right)^{\otimes 3}$. Thus the associated representation of $S^{1}$ is given by $K r^{3} \circ h_{\lambda}$, where $h_{\lambda}$ denotes the Hodge structure of $\mathcal{E}_{\lambda}$. Therefore the sub-Hodge structure on $\operatorname{Sym}^{3}\left(H^{1}\left(\mathcal{E}_{\lambda}, \mathbb{Q}\right)\right)$ is given by

$$
h^{\prime}=r \circ h_{\lambda} .
$$

One concludes $h^{\prime}\left(S^{1}\right) \subset G_{\mathbb{R}}$, since $h_{\lambda}\left(S^{1}\right) \subset \mathrm{SL}\left(H^{1}(E, \mathbb{R})\right)$ and $r$ yields a homomorphism $\mathrm{SL}\left(H^{1}(E, \mathbb{R})\right) \rightarrow G_{\mathbb{R}}$.

Proposition 4.13. The variation $\mathcal{V}$ of Hodge structures is of third type.

Proof. Since $h^{\prime}\left(S^{1}\right) \subset G_{\mathbb{R}}$, the conjugation by $h^{\prime}(i)$ yields a Cartan involution of $G_{\mathbb{R}}$. Thus $G$ is reductive. Since $\operatorname{dim} G \leq 3$, this group is not only reductive, but simple. This follows from the fact that the smallest simple Lie algebras have dimension 3 and $G$ is clearly not commutative. Therefore the center of $G_{\mathbb{R}}$ is discrete the associated hermitian symmetric domain is $\mathbb{B}_{1}$. Hence $\mathcal{V}$ is of third type.

Acknowledgements. This paper was written at the Graduiertenkolleg "Analysis, Geometry and Stringtheory" at Leibniz Universität Hannover. I would like to thank Klaus Hulek for his interest and his comments, which helped to improve this text. Moreover I would like to thank Martin Möller for a fruitful discussion and Eckart Viehweg for the hint to [13], which helped to find a Shimura curve with a Calabi-Yau like $V H S$ of third type.

\section{REFERENCES}

[1] C. Borcea, Calabi-Yau threefolds and complex multiplication, Essays on mirror manifolds, Internat. Press, Hong Kong (1992), pp. 489-502.

[2] R. Bryant and P. Griffiths, Some Observations on the Infinitesimal Period Relations for Regular Threefolds with Trivial Canonical Bundle, in: Arithmetic and Geometry II, Progress in Mathematics 36, Birkhäuser, Boston Basel Stuttgart (1983), pp. 77-102.

[3] P. Deligne, Travaux de Shimura. In: Seminaire Bourbaki, 389 (1970/71), Lecture Notes in Mathematics 244, Springer-Verlag, Berlin (1971), pp. 123-165.

[4] P. Deligne, Variétés de Shimura: interprétation modulaire, et techniques de construction de modèles canoniques, in: Automorphic forms, representations and $L$-functions (Proc. Sympos. Pure Math., Oregon State Univ., Corvalis, Ore., 1977), Part 2, Proc. Sympos. Pure Math., XXXIII, AMS, Providence, R.I. (1979), pp. 247-289.

[5] C. Doran and J. Morgan, Mirror Symmetry and Integral Variations of Hodge Structure, Stud. in adv. Math., 38 (2006), pp. 517-538.

[6] S. Helgason, Differential Geometry, Lie Groups, and Symmetric Spaces, Graduate Studies in Mathematics, 34, AMS (2001) Providence, RI.

[7] J. S. Milne, Introduction to Shimura Varieties, preprint (2004).

[8] D. Morrison, Compactifications of moduli spaces inspired by mirror symmetry, Journées de géométrie algébrique d'Orsay, Astérisque, 218 (1993), pp. 243-271.

[9] B. Moonen, Linearity properties of Shimura varieties, Part I, J. Algebraic Geom., 7 (1998), pp. 539-567.

[10] J. C. Rohde, Cyclic coverings, Calabi-Yau manifolds and Complex multiplication, Lecture Notes in Mathematics 1975, Springer-Verlag (2009) Berlin, Heidelberg.

[11] J. C. RoHde, Maximal automorphisms of Calabi-Yau manifolds versus maximally unipotent monodromy, Manuscripta Math., 131 (2009), pp. 459-474

[12] I. Satake, Algebraic structures of symmetric domains, Kanô Memorial Lectures 4, Iwanami Shoten, Princeton University Press (1980) USA.

[13] E. Vienweg And K. Zuo, Families over curves with a strictly maximal Higgs field, Asian J. Math., 7 (2003), pp. 575-598.

[14] C. Voisin, Théorie de Hodge et géométrie algébrique complexe, Cours spécialisés 10, SMF (2002) France.

[15] Y. ZARKHIN, Weights of simple Lie algebras in the cohomology of algebraic varieties, Math. USSR-Iz., 24 (1985), pp. 264-304. 
J. C. ROHDE 